\title{
Account
}

\section{Coherent Two-Dimensional Optical Spectroscopy}

\author{
Minhaeng Cho \\ Deparment of Chemistry and Center for Multidimensional Spectroscopv, Korea Lniversitv, Seoul 136-701, Korea \\ E-mail: moholakoreack $k$ \\ Received June 23, 2006
}

\begin{abstract}
Theoretical descriptions of two-dimensional (2D) vibrational and electronic spectroscopy are presented. By using a coupled multi-chromophore model. some examples of 2D spectroscopic studies of peptide solution structure determination and excitation transfer process in electronically coupled multi-chromophore ș'stem are discussed. A few remarks on perspectives of this research area are given.
\end{abstract}

Key Words : Two-dimensional spectroscopy. Molecular dỵnamics. Protein dỵnanics

\section{Introduction}

Two-dimensional (2D) optical spectroscopy' utilizing multiple ultrafast coherent laser pulses have been used to study protein structure and dynamics ${ }^{1-7}$ femtosecond solvation dy'namics. ${ }^{8}$ hy'drogen-bonding dynamics. ${ }^{y-12}$ solute-solvent complexation. ${ }^{13}$ and excitation migration process in a photosynthetic light harvesting complex. ${ }^{14}$ Due to dramatic advent of recent laser teclunology. femtosecond laser sy'stems operating in infrared and visible frequency range have been commercially available so that we have seen a wide range of applications utilizing such ultrafast nonlinear spectroscopic tecluniques.

Most of the conventional linear spectroscopies. though they are still extremely' useful for studying structural and dynamic properties of complex molecules. can only provide highly averaged information. Therefore novel spectroscopic tecluniques with much higher information content has been sought and tested continuously. In NMR spectroscopy. such efforts led to developing a variety of $2 \mathrm{D}$ NMR techuiques such as NOESY (Nuclear Overhauser Enhancement Spectroscopy) and COSY (conrelation spectroscopy) methods among many others and they have been extensively used to study' structural and dynamical properties of proteins in solution. ${ }^{15-j 7}$

In Figure 1. a direct analogy between 2D vibrational spectroscopy and 2D NMR is depicted. In the case of 2D NMR. one can measure the nuclear spin-spin coupling constant that carries information on the 3D molecular structure. Similarly, the 2D vibrational spectroscopy can provide

Minhaeng Cho is a Professor of Chemistry at Korea University and Director of the Center for Multidimensional Spectroscopy. He received his B.S. and M.S. degrees in chemistry from the Seoul National University in Seoul. Korea. He then moved to the University of Chicago where he earned his Ph.D. in Physical Chemistry from Prof. G. R. Fleming in 1993. After receiving post-doctoral training at MI. Cambridge. U.S.A. with Prof. R. J. Silbey in the field of theoretical physical chemistry of nonlinear optical spectroseopy and electron transfer processes, he joined the Korea University as an assistant professor in

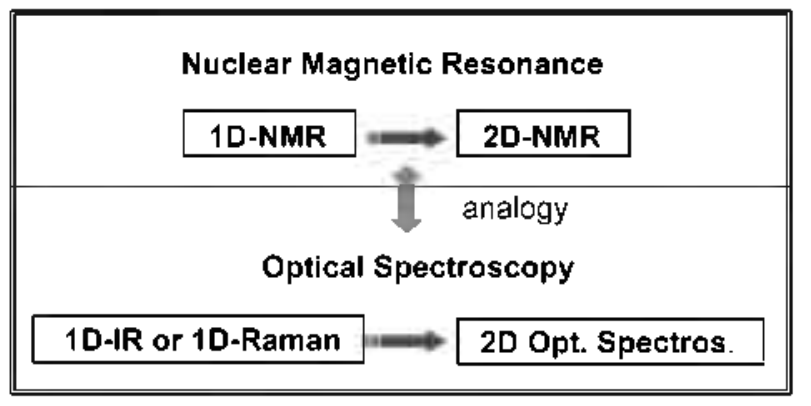

Figure 1. An analogy between two-dimensional NMR and twodimensional optical spectroscopies.

information on the vibrational coupling constant between two spatially separated vibrational modes and the 2D electronic spectroscopy has been found to be useful for estimating the magnitudes of electronic coupling constant between two optical chromophores. Despite that the interaction mechanism between two different vibrational (electronic) degrees of freedom differs from that between two nuclear spins. the coupling strengths in both cases are sensitively dependent on the 3D molecular structure (Fig. 2).

Although the optical analogs of 2D NMR do not provide an atomic resolution structure of complex molecules. optical domain multi-dimensional spectroscopy has certain advantages because of the dramatic gain in time resolution ( $\sim$ subpicosecond scale) possible and because of the ability to directly observe and quantify the couplings between quantum states involved in molecular dynamical processes. For the

1996 and became a protessor in 2002 . He was elected as a junior member of the Korean Academy of Science and Technology in 2002. He is a recipient of the Nobel Laureate Signatures A ward (American Chemical Society) in 1995. Young Scientist A ward (Ministry of Science and Technology. Korea) in 1999. British Chevening Award in 2000). and now is a Hy undai-Kia Motor Professor of Korea University since 2005 . His research areas include the nonlinear optical spectroscopy and molecular dynamies and computational chemistry of biomolecules in condensed phases. 


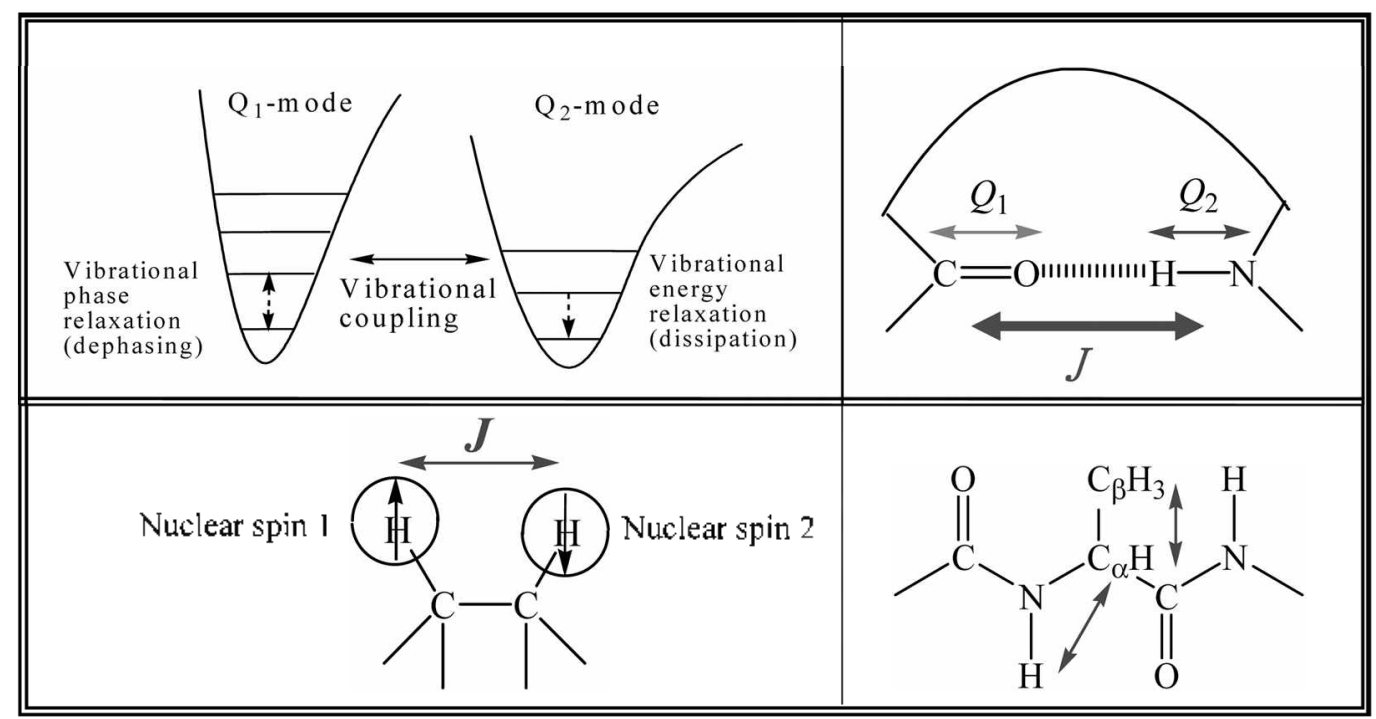

Figure 2. NMR spectroscopy can provide stucture information by measuring the coupling constant between two nuclear spins. Similarly, the two-dinensional vibrational spectroscopy is useful in extracting quantitative information on the vibrational coupling constant between two local oscillators.

2D vibrational spectroscopy. an elementary and highly simplified schematic diagram in Figure 3 shows the reason why 2D vibrational spectroscopic technique can provide detailed information on the 3D structure of a given complex molecule. The existence of cross peaks can be direct evidence on the vibrational coupling induced by the spatial proximity between the two different vibrational chromophores in this case. Similarly. if two optical chromophores are close to each other the electronic coupling between the two induces an electronic exciton fomation and produces cross peaks in the $2 \mathrm{D}$ electronic spectrum. ${ }^{18,13}$ Therefore, experimental observation of cross peaks in a measured 2D electronic spectrum can be considered to be direct evidence of the electronic coupling between two chromophores. In the present paper. we will mainly focus on our recent theoretical studies of $2 \mathrm{D}$ vibrational as well as electronic spectroscopies

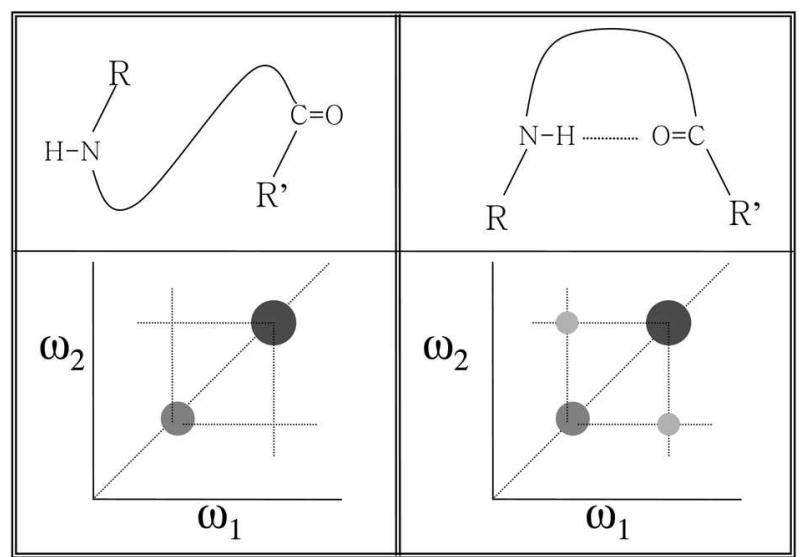

Figure 3. A schematic figure showing that the existence of cross peaks in a two-dimensional vibrational spectroscopy is direct evidence on the spatial proxinnity of the two vibrational chromophores. of coupled multi-chromophore systems.

It should be mentioned that the main goal of this article is not to review the entire experimental and theoretical works reported in this research area but to provide brief accounts of works mostly presented by the author's group. Therefore, for those who are interested in reading other reviews. references 2-7 and 18 could be of great interest. The present paper is organized as follows. In Sec.II, a general description of ultrafast vibrational or electronic excitation and probing methods utilizing femtosecond laser pulses is discussed. The linear and nonlinear response functions for any arbitrary multi-level systems are theoretically described by using the correlation function theory. In Sec.IV the theoretical model Hamiltonians for coupled multi-cluromophore sy'stems consisting of either two-level cluromophores or anharmonic oscillators are discussed and a variety of auxiliary functions such as frequency-frequency correlation functions. transition dipole matrix elements. etc. are summarized. A variety of 2D vibrational spectroscopic studies of the prototype monopeptide sy'stem, dipeptide. $\alpha$-helix, $\beta$-hairpin, and extended multiple-stranded $\beta$-sheet polypeptides are discussed in Sec.V. A recent application of $2 \mathrm{D}$ electronic spectroscopic method to studying excitation migration process in a photosy'nthetic light-harvesting complex is briefly discussed in Sec.VI. A few future applications and perspectives of 2D optical spectroscopy and a concluding remark are given in Sec.VII.

\section{Ultrafast Excitation and Probing Methods}

In order to classify each different coherent multidimensional spectroscopic teclnique. it is necessary to describe how they differ from one another at the level of optical excitation and probing methods.

A. Ultrafast excitation methods. There are numerous 
time-donain methods to vibrationally or electronically excite molecules in condensed phases. Depending on the molecular nonlinear response of interest. one can probe the relaxation of the diagonal (population state) or off-diagonal (coherent state) density matrix elements selectively. ${ }^{\hat{i}^{i}}$ Thus. the ultrafast excitation methods can be divided into two classes. Note that the former ty'pe of experiment is to measure time-dependent population relaxation process by stimulating emission with an ultrafast laser pulse or by detecting spontaneously emitted field intensity. The latter type of spectroscopy is useful in measuring vibrational or electronic decoherence rate. In Figure 4. we specifically consider five different vibrational excitation methods.

(E1) Absorption of a resonant IR photon

(E2) Stimulated Raman excitation by electronically nonresonant optical fields

(E3) Stimulated hyper-Raman excitation by electronically non-resonant optical fields

(E4) Absorption of a resonant circularly polarized IR photon

(E5) Stimulated Raman excitation by electronically nonresonant (circularly polarized) optical fields.

One of the most straightforward methods to create a vibrational coherence state is to use a resonant infrared pulse. The IR-field-matter interaction is. within the electric dipole approximation. $-\mu E(t)$ so that the force exerted on the $j$ th vibrational degree of freedom is $-\left(\partial \mu / \partial Q_{j}\right) E(t)$, where $\mu$ is the electric dipole moment. Due to the finite spectral bandwidth of femtosecond IR pulse. $\ddot{E}(\omega)$. which is the Fourier-transform of the temporal envelope of the IR pulse. the excitation process involves a range of vibrational excitations of modes of which frequencies are within the envelope of the pulse spectrum. $\ddot{E}(\omega)$. The second method (E2) is to use stimulated Raman process to vibrationally excite the electronically ground state molecule. By using an ultrafast optical pulse whose frequency is electronically non-resonant. Raman-active vibrational modes can be excited when the corresponding vibrational frequencies are within the spectral bandwidth of the short pulse. In this case. the effective field matter interaction is approximately given as $-\alpha|E(t)|^{2}$. where

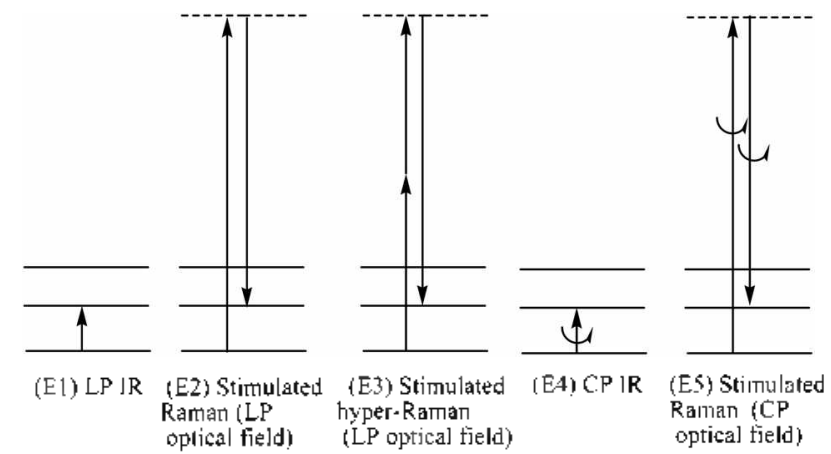

Figure 4. Five representative vibrational coherence state generation methods. Horizontal lines are vibrational quantum states on the electronic ground state. Dashed horizontal line corresponds to a virtual state involved in the Rannan or hyper-Raman scattering processes. 'The methods $\mathrm{E} 4$ and $\mathrm{E} 5$ involve circularly polarized IK and electronically non-resonant optical tields, respectively. $\alpha$ is the molecular polarizability. so that the force exerted on the $j$ th vibrational degree of freedom is $\left(\partial \alpha / \partial Q_{2}\right)|E(t)|^{\text {. }}$. Various stimulated Raman scattering experiments involve such kind of vibrational excitation processes. The third method (E3) involves higher-order field-matter interaction. the hyper-Raman process. so that the effective field-matter interaction in this case is $-\beta E_{1}(t) E_{\widehat{\alpha}}(t) E_{3}^{*}(t)$, where $\beta$ is the first molecular hyperpolarizability and three injected fields are denoted as $E_{l}(t)$. Then. the $j$ th mode experiences the force. $\left(\partial \beta / \partial Q_{l}\right) E_{1}(t) E_{2}(t) E_{3}^{*}(t)$. when the molecule is exposed to these electronically off-resonant fields. Often, the vibrational selection rule of the hyper-Raman process differs from that of the Raman process so that the stimulated hyperRaman excitation method can be of use to excite different vibrational degrees of freedom in comparison to the conventional impulsive stimulated Raman scattering method. The first three methods utilize linearly polarized beams. but one can employ circularly polarized (CP) beams and measure the difference between the left-CP and right-CP signals to extract information on the molecular optical activity. As theoretically proposed recently. time-resolved optical activity measurement can be achieved by using CP light beanıs in the sum-frequency-generation or four-wave-mixing spectroscopic schemes. ${ }^{11-23}$ These novel spectroscopic methods can be of effective use in studying ultrafast dynamics of chiral molecules in condensed phases. The fourth and fifth methods. E4 and E5 in Figure 3. utilize CP IR and CP optical pulsed fields to create a vibrational coherence state.

B. Ultrafast probing methods. Time-dependent evolution of thus created vibrational coherence states can be probed by employing suitable ultrafast probing methods. Much like the ultrafast excitation (vibrational coherence state generation) process. one can use the same types of coherent transition processes. In Figure 5. the energy' level diagrams associated with the five spontaneous or stimulated emission detection methods are shown.

The first method $(\mathrm{Pl})$ is to measure the spontaneously emitted IR field by homodyne or heterodyne-detection method. The P2 method uses the stimulated Raman scattering process. Suppose that a vibrational coherence state is

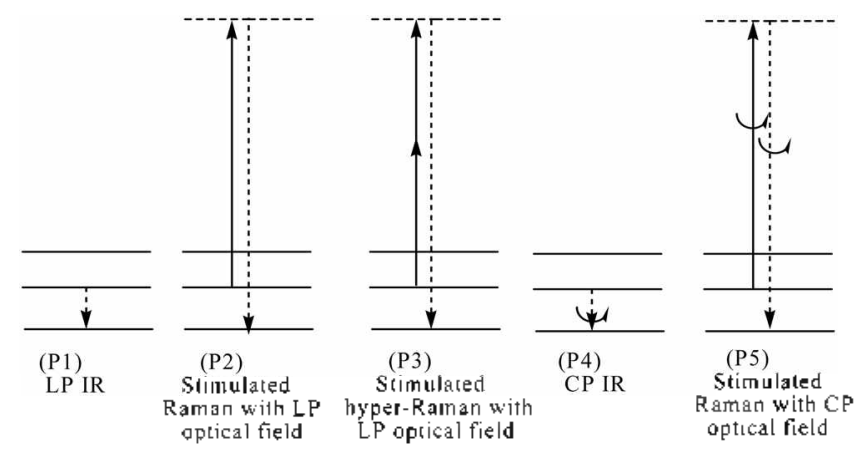

Figure 5. The upward solid arrow represents transition induced by extemal-field-matter interaction, whereas the downward dashed arrow is spontaneously emissive field-matter interaction. The tourth and fith probing methods, 44 and $P_{5}$, involve detection of circularly polarized component of signal electric tield. 
created by preceding field-matter interactions. Then. an electronically off-resonant optical field can be inelastically scattered by this vibrational transient grating and the measured signal intensity or amplitude contains information on the evolution of the vibrational coherence state i.e. linear or nonlinear vibrational response of the molecular sy stem. The third method (P3) is to use higher-order (hy'perRaman) scattering process to probe dynamic evolution of the vibrational coherence state. The other two methods. $\mathrm{P} 4$ and P5. are self-evident.

C. Designing a coherent multidimensional spectroscopy. Using one of the schemes in Figure 4. one can create a ID transient grating (TG) in a given optical sample. Depending on the probing method in combination with a chosen excitation method. one can measure a variety of different molecular properties such as dipole polarizability. or hyperpolarizability correlation functions. For instance. let us consider the coherent Raman scattering spectroscopy utilizing femtosecond laser pulses. For the $j$ th vibrational coherence state. its relaxation can be approximately described as $e^{\gamma / f} \sin \omega_{j} t$. where $\gamma_{3}$ is the vibrational dephasing constant. After a finite delay time. the transient dynamics of this 1D grating can be measured by using one of the time-domain probing methods in Figure 5. Týpical one-dimensional spectroscopy such as absorption. Raman scattering. and other related tecluniques. is to measure the oscillation frequency and dephasing rate of this transient grating. i.e.. linear polarization

Then. by combining two or more excitation pulses that are separated in time. it will be possible to create temporally' (and/or spatially) multi-dimensional vibrational (or electronic) TG's. Unlike the ID TG the signal field generated by the nonlinear polarization reflecting dynamic evolution of the multi-dimensional TG is produced by the interference between electric fields created by different nonlinear optical transition pathways. The two- and three-dimensional spectroscopies are essentially to measure the temporal evolution and relaxation of thus created two- and three-dimensional transient gratings. In practice. one measures the two- or three-dimensional signal. $S\left(t_{1}, t_{2}\right)$ or $S\left(t_{1}, t_{2}, t_{3}\right)$, in time domain and its multi-dimensional Fourier transformation gives the corresponding $2 \mathrm{D}$ or $3 \mathrm{D}$ spectnum.

As outlined above. there are different ways to create coherent states. Consequently. there could be a number of different ways to create 2D TG However only a few of them have been experimentally explored so far. In Scheme 1. we show some combinations of excitation and probing methods. If the first three methods in Figure 4 are only'
$\Theta$

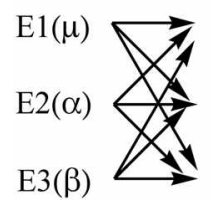

$\Lambda$

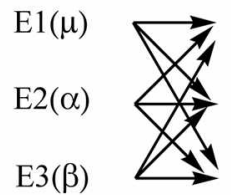

$\Phi$

$\mathrm{P} 1(\mu)$

$\mathrm{P} 2(\alpha)$

$\mathrm{P} 3(\beta)$
$\Theta$

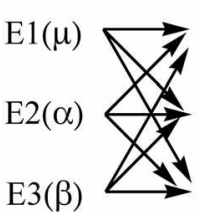

$\Lambda$
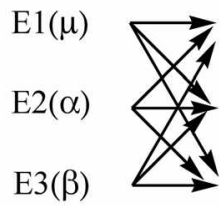

E1 $(\mu)$
E2 $(\alpha)$
E3( $\beta)$

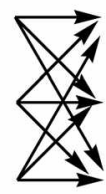

$\operatorname{P} 1(\mu)$

$\mathrm{P} 2(\alpha)$

$\mathrm{P} 3(\beta)$

considered. there are 9 distinctively different ways to create a $2 \mathrm{D}$ TGi.e.

Then. the corresponding nonlinear response function is defined as

$$
R^{(\hat{2})}\left(t_{2}, t_{1}, t_{i j}\right)=\left(\frac{i}{h}\right)^{\hat{\imath}}\left\langle\left[\left[\Phi\left(t_{2}\right) \cdot \Lambda\left(t_{1}\right)\right] \cdot \Theta\left(t_{0}\right)\right] \rho\left(t_{i j}\right)\right\rangle .
$$

where the thermal equilibrium density operator was denoted as $\rho\left(t_{0}\right)$.

In the case of three-dimensional spectroscopy, there are 27 different way's to create 3D transient grating (see Scheme 2).

The third-order nonlinear response function describing molecular response to a specific sequence of field-matter interactions in the above scheme is defined as

$$
\begin{aligned}
& R^{\left(\pi_{1}\right.}\left(t_{3}, t_{2}, t_{1}, t_{0}\right) \\
& \quad=\left(\frac{i}{n}\right)^{2}\left\langle\left[\left[\left[\Psi\left(t_{3}\right) \cdot \Phi\left(t_{2}\right)\right] . \Lambda\left(t_{1}\right)\right], \Theta\left(t_{0}\right)\right] \rho\left(t_{1}\right)\right\rangle .
\end{aligned}
$$

where $\Psi, \Phi . \Lambda$ and $\Theta$ are molecular operators. such as dipole $(\mu)$. polarizability $(\alpha)$. first hyperpolarizability $(\beta)$ etc.

For example. the photon echo spectroscopy generally involves three or four laser pulses that are separated in time and its field-matter interaction sequence is given as E1-ElEl-Pl. ${ }^{24} 25$ The funal pulse in the case of the heterodynedetected photon echo experiment acts like a time-gating pulse. Thus. the photon echo technique belongs to the case of 3D TG experiment in general. However. if. during the second delay time $t$. the system is on the population state (diagonal density matrix),there are two coherence state evolution periods so that the photon echo method can be considered to be one of the coherent 2D spectroscopy i.e. a reduced 2D vibrational spectroscopy ${ }^{6}$

Now, the next step toward the complete theoretical description of the coherent multi-dimensional spectroscopy is to obtain the corresponding nonlinear response function. which is required in the calculation of nonlinear polarization and signal electric field.

\section{Linear and Nonlinear Response Functions}

In the present section, we will provide theoretical expressions of linear and nonlinear response functions given in Eqs.(1) and (2). Note that the nth-order polarization can be written as ${ }^{\text {26 }}$

$$
\mathbf{P}^{(n)}(\mathbf{r}, t):=N \int_{10}^{\infty} d t_{n} \cdots \int_{0}^{\infty} d t_{2} \int_{10}^{\infty} d t_{1} R^{i n 1}\left(t_{n} \cdots, t_{2} \cdot t_{1}\right)
$$




$$
\times \mathbf{E}\left(\mathbf{r} \cdot t-t_{n}\right) \mathbf{E}\left(\mathbf{r} \cdot t-t_{n-1}-t_{n-1}\right) \times \cdots \times \mathbf{E}\left(\mathbf{r}, t-t_{n}-\cdots t_{2}-t_{1}\right)
$$

where $N$ is the number of chromophores. The measured $n$ thorder signal field amplitude is related to the $n$ th-order polarization as $\mathbf{E}^{(n)}: j \omega \mathbf{P}^{i n !}$. Now by using the eigenstate representation and taking into account the system-bath interaction properly. the linear and nonlinear response functions can be obtained and written as $27-54$

$$
\begin{aligned}
& R^{i l \prime}\left(t_{1}\right)=\left(\frac{i}{h}\right) \theta\left(t_{1}\right) \sum_{a b} P_{a}\left(t_{0}\right) \\
& \times\left\{\Lambda_{a b b} \Theta_{b_{b}} \exp \left(-i \omega_{b_{b} t_{1}}\right) F_{b_{c} a}\left(t_{1}\right)-c . c\right\} \\
& R^{(2)}\left(t_{2}, t_{1}\right)=\left(\frac{i}{h}\right)^{2} \theta\left(t_{2}\right) \theta\left(t_{1}\right) \sum_{a b c} P_{a}\left(t_{0}\right) \\
& \times\left[\Phi_{a b} \Lambda_{b c} \Theta_{c a} \exp \left(-i \omega_{b a} t_{2}-i \omega_{c a} t_{1}\right) G_{b a}^{i !}\left(t_{2}, t_{1}\right)\right. \\
& \left.-\Lambda_{c i b} \Phi_{b c} \Theta_{c i b} \exp \left(-i \omega_{c b} t_{2}-i \omega_{c a} t_{1}\right) G_{b i l}^{(2)}\left(t_{2} \cdot t_{1}\right)\right]+c . c \\
& R^{(3)}\left(t_{3}, t_{2}, t_{1}\right)=\left(\frac{i}{h}\right)^{3} \theta\left(t_{1}\right) \theta\left(t_{2}\right) \theta\left(t_{3}\right) \sum_{\alpha=1}^{+}\left[H_{\alpha}\left(t_{3}, t_{2}, t_{1}\right)-c . c .\right] \text {, }
\end{aligned}
$$

where the auxiliary functions are

$$
\begin{aligned}
& F_{b a}\left(t_{1}\right)=\exp \left\{-\int_{i j}^{t_{1}} d \tau_{2} \int_{i j}^{\tau_{2}} d \tau_{1} \xi_{b b}\left(\tau_{2}-\tau_{1}\right)\right\} \\
& G_{b c}^{(1)}\left(t_{1}, t_{2}\right)=\exp \left\{-\left[\int_{0}^{t_{2}} d \tau_{1} \int_{1 j}^{\tau_{1}} d \tau_{2} \xi_{b b}\left(\tau_{1}-\tau_{2}\right)\right.\right. \\
& \left.\left.+\int_{0}^{t_{1}} d \tau_{1} \int_{0}^{\tau_{1}} d \tau_{2} \xi_{c c}\left(\tau_{1}-\tau_{2}\right)+\int_{0}^{t_{2}} d \tau_{1} \int_{0}^{t_{1}} d \tau_{2} \xi_{b c}\left(\tau_{1}+\tau_{2}\right)\right]\right\} \\
& G_{b c}^{(2)}\left(t_{1}, t_{2}\right)=\exp \left\{-\left[\int_{0}^{t_{2}} d \tau_{1} \int_{1 j}^{\tau_{1}} d \tau_{2} \xi_{b b}^{*}\left(\tau_{1}-\tau_{2}\right)\right.\right. \\
& \left.\left.+\int_{01}^{t^{2} \tau_{2}} d \tau_{1} \int_{i j}^{\tau_{1}} d \tau_{3} \xi_{c c}\left(\tau_{1}-\tau_{2}\right)+\int_{i j}^{t_{2}} d \tau_{1} \int_{01}^{t_{0} \tau_{2}} d \tau_{2} \xi_{b c}\left(\tau_{1}-\tau_{2}\right)\right]\right\}
\end{aligned}
$$

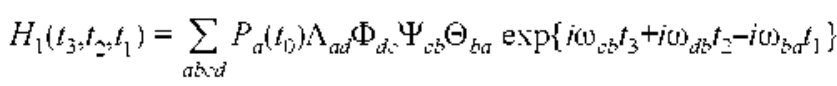

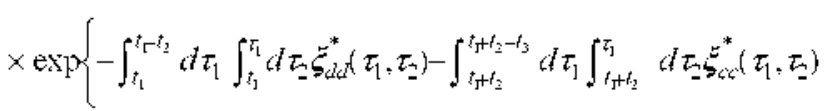

$-\int_{0}^{t_{1} t_{2}+t_{3}} d \tau_{1} \int_{10}^{\tau_{1}} d \tau_{\Sigma} \xi_{z b}\left(\tau_{1}, \tau_{2}\right)-\int_{t_{1}}^{t_{1}+t_{2}} d \tau_{1} \int_{t_{\Gamma} t_{2}}^{t_{r_{2}}-t_{3}} d \tau_{\Sigma} \zeta_{d i}\left(\tau_{1}, \tau_{2}\right)$

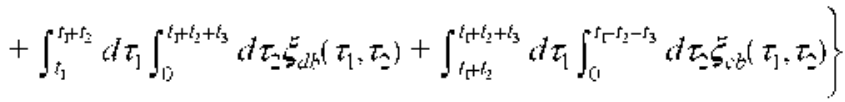

$H_{z}\left(t_{3}, t_{2}, t_{1}\right)=\sum_{a b v d} P_{a}\left(t_{10}\right) \Theta_{a d} \Phi_{d k} \Psi_{v b} \Lambda_{b a} \exp \left\{j \omega_{c b} t_{3}+j \omega_{d b} t_{3}-i \omega_{b \sigma} t_{1}\right\}$

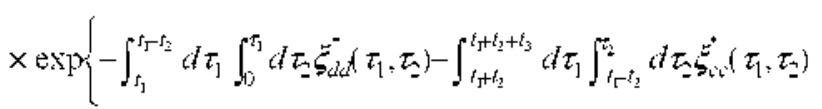

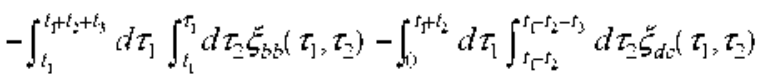

$\left.+\int_{0}^{t_{c} t_{3}} d \tau_{1} \int_{t_{1}}^{t_{1}+t_{2}-t_{3}} d \tau_{2} \xi_{d k}\left(\tau_{1}, \tau_{2}\right)+\int_{t_{1}+t_{2}}^{t_{t}+t_{2}-t_{3}} d \tau_{1} \int_{t_{1}}^{t_{1}+t_{2}+t_{3}} d \tau_{2} \xi_{c k}\left(\tau_{1}, \tau_{2}\right)\right\}$

$H_{3}\left(t_{3}, t_{2}, t_{1}\right)=\sum_{a b u d} P_{d}\left(t_{i}\right) \Theta_{u d} \Lambda_{d i} \Psi_{i b}^{\prime} \Phi_{h u} \exp \left\{i\left(\omega_{c h} t_{3}+i \omega_{c d} t_{2}+i \omega_{d u} t_{1}\right\}\right.$

$\times \exp \left\{-\int_{i}^{t_{1}} d \tau_{1} \int_{i}^{\tau_{1}} d \tau_{2} \xi_{d d}^{*}\left(\tau_{1}, \tau_{2}\right)-\int_{t_{1}}^{t_{1}+t_{2}-t_{3}} d \tau_{1} \int_{t_{1}}^{\tau_{3}} d \tau_{-} \xi_{c o}^{*}\left(\tau_{1}, \tau_{2}\right)\right.$

$-\int_{\tau_{\tau} \tau_{2}}^{T_{\tau} \tau_{2}-\tau_{3}} d \tau_{1} \int_{\tau_{1}+t_{2}}^{\tau_{1}} d \tau_{2} \xi_{h b}\left(\tau_{1}, \tau_{2}\right)-\int_{1_{1}}^{\tau_{1}} d \tau_{1} \int_{\tau_{1}}^{t_{1}+t_{2}+t_{3}} d \tau_{2} \xi_{d i}\left(\tau_{1}, \tau_{3}\right)$

$\left.+\int_{01}^{T_{1}} d \tau_{1} \int_{T_{1}+t_{2}}^{T_{1}+t_{2}-t_{3}} d \tau_{2} \xi_{d} d\left(\tau_{1}, \tau_{2}\right)+\int_{T_{1}}^{T_{1}+t_{2}-t_{3}} d \tau_{1} \int_{t_{1}+t_{2}}^{t_{1}+t_{2}+t_{3}} d \tau_{\Sigma} \xi_{23}\left(\tau_{1}, \tau_{2}\right)\right\}$

$H_{4}\left(t_{3}, t_{2} t_{1}\right)=\sum_{a b v d} P_{a}\left(t_{0}\right) \Psi_{a d} \Phi_{d k} \Lambda_{c b} \Theta_{b a} \exp \left\{-i \omega_{d a} t_{3}-i \omega_{v a} t_{2}-i \omega_{z a} t_{1}\right\}$

$\times \exp \left\{-\int_{T_{\Gamma} \tau_{2}}^{T_{\Gamma} \tau_{2}-\tau_{3}} d \tau_{1} \int_{T_{1}+t_{2}}^{\tau_{1}} d \tau_{2} \xi_{2 d} d \tau_{1}, \tau_{-}\right)-\int_{t_{1}}^{t_{1} t_{2}} d \tau_{1} \int_{\tau_{1}}^{\tau_{1}} d \tau_{2} \xi_{3}\left(\tau_{1}, \tau_{2}\right)$

$-\int_{01}^{t_{1}} d \tau_{1} \int_{10}^{\tau_{1}} d \tau_{3} \xi_{h b}\left(\tau_{1}, \tau_{2}\right)-\int_{t_{1}+t_{2}}^{t_{1}+t_{2}-t_{3}} d \tau_{1} \int_{t_{1}}^{t_{1}+t_{2}} d \tau_{3} \xi_{d i}\left(\tau_{1}, \tau_{2}\right)$

$\left.-\int_{t_{5} t_{2}}^{t_{5} t_{2}-t_{3}} d \tau_{1} \int_{10}^{t_{1}} d \tau_{3} \xi_{d 3}\left(\tau_{1}, \tau_{2}\right)-\int_{t_{1}}^{t_{1} t_{2}} d \tau_{1} \int_{a_{1}}^{t_{1}} d \tau_{3} \xi_{23}\left(\tau_{1}, \tau_{2}\right)\right\}$.

Here, $P_{a}\left(t_{0}\right)$ is the Bolzmann probability of finding the quantum state $|a\rangle$ at time $t \rightarrow$ at temperature $T$. The complex conjugate is denoted as c.c. $\theta(t)$ is the Heavyside step function. The transition matrix element. for example $\Theta_{3 a \text { a }}$ is defined as $\Theta_{3 a}=\langle b|\Theta| a\rangle$. The entire response functions are detemined by the complex auto- and cross-correlation functions of the fluctuating transition frequencies defined as

$$
\xi_{x y}\left(\tau_{1}, \tau_{-}\right) \equiv\left\langle\delta \omega_{x \mathrm{x}}\left(\tau_{1}\right) \delta \omega_{y x}\left(\tau_{-}\right)\right\rangle .
$$

The linear and nonlinear response functions can be expressed in terms of the correlation functions of the bath degrees freedom that are coupled to the molecular vibrational or electronic transition.

\section{Coupled Multi-Chromophore Model}

Both polypeptides and molecular complexes with a number of vibrational or electronic cluromophores that are coupled to each other electronically can be successfully modeled by using the Frenkel exciton model. where each monomeric chromophore is either a two-level system or an anharmonic oscillator system. In the case of the amide I vibrational modes, each individual amide I local oscillator can be approximated as an anharmonic oscillator with just three low-lying vibrational states. Note that most of the 2D vibrational spectroscopic techniques based on the fourwave-mixing method such as $2 \mathrm{D}$ IR punp-probe and photon echo involve vibrational transitions up to the second excited states that are either overtone or combination states. Thus, one can assume that a vibrational chromophore in this case is a three-level system with the overtone anhammonicity correctly taken into consideration. On the other hand. for coupled multi-chromophore system such as photosynthetic 
light-harvesting complex or $J$-aggregates. where the associated optical transition is electronic in nature. one can assume that the monomer is nothing but a two-level sy'stem. However. due to the electronic couplings among the two-level monomeric chromophores. the entire multi-chromophore system consists of not only the one-exciton states but also the two-exciton states. Nevertheless the model Hamiltonians for these two different classes of complicated molecular systems are little different from each other.

A. Model Hamiltonian for coupled multi-chromophore systems. In order to describe nonlinear optical properties of molecular complexes and aggregates. the Frenkel exciton Hamiltonian has been extensively used ${ }^{3 j}$ Denoting $a_{m}^{-}$and $a_{m}$ to be the creation and anniluiation operators of an electronic excitation at the $m^{\text {th }}$ clromophore, the zero-order Hamiltonian can be written as

$$
H_{01}=\sum_{n=1}^{N} \hbar\left(i_{m} a_{m} a_{m}+\sum_{m}^{N} \sum_{n \neq m}^{N} \hbar J_{m m} a_{m} a_{m}+H_{p h}\right.
$$

where the excited state energy of the $m^{\text {th }}$ chromophore, electronic coupling constant between the $m^{\text {th }}$ and $n^{\text {th }}$ cluromophores. and the phonon bath Hamiltonian were denoted as $\hbar(1)_{m}, J_{m m}$ and $H_{p n^{\prime}}$ respectively. If each monomeric chromophore is an anharmonic oscillator instead of a twolevel system. the zero-order Hamiltonian should be written as

$$
\begin{aligned}
H_{0}= & \sum_{m=1}^{N} \hbar(0)_{m} a_{m}^{+} a_{m}+\sum_{m}^{N} \sum_{n \neq m}^{N} \hbar J_{m m} a_{m}^{-} a_{m s} \\
& +\sum_{n}^{N} \sum_{n}^{N} \hbar \Delta_{m m n} a_{m}^{+} a_{n t}^{+} a_{m} a_{n t}+H_{p h f}
\end{aligned}
$$

Note that the third term on the right-hand side of Eq describes the quartic anharmonicities.

The chromophore-bath interaction and the changes of inter-chromophore distance and orientation induce fluctuations of site energies and coupling constants. Thus the general system-bath interaction Hamiltonian is written as

$$
H_{S B}=\sum_{m} \sum_{n} \hbar q_{m n}(\mathbf{Q}) a_{m}^{-} a_{n}
$$

where $q_{m u}(\mathbf{Q})$ is an operator of bath coordinates. $\mathbf{Q}$. and it is assumed that the expectation values calculated over the bath eigenstates. $\left\langle q_{m n}(\mathbf{Q})\right\rangle_{1}$. are zero-note that if $\left\langle q_{m n}(\mathbf{Q})_{0_{1}}\right.$ values are finite they can be included in the zero-order Hamiltonian. For $m \neq n$. the couplings to bath degrees of freedom can induce excitation transfers between different chromophores in the site representation. The total Hamiltonian can therefore be written as

$$
H=H_{0}+H_{S B}+H_{B}
$$

B. Transformation into delocalized exciton representation. For any general four-wave-nixing spectroscopy, we need to consider three well-separated quantum state manifolds: the ground state. $N$ one-exciton states and $\sim N^{2}$ twoexciton states. The one- and two-exciton eigenvalues and eigenvectors can be obtained by diagonalizing the one- and two-exciton Hamiltonian matrices obtained in the site representation. and these two matrices are denoted as $\tilde{H}_{l}$ and $\tilde{H}_{2}$. Then we have

$$
\begin{aligned}
& U^{-1} \tilde{H}_{1} U=\hbar \tilde{\Omega} \\
& V^{-1} \tilde{H}_{2} V=\hbar \tilde{W} .
\end{aligned}
$$

where the one- and two-exciton eigenvalues are the diagonal matrix elements of $\hbar \tilde{\Omega}$ and $\hbar \tilde{W}$. respectively. The one- and two-exciton states are therefore linear combinations of singly or doubly excited states wavefunctions, i.e.

$$
\begin{aligned}
& \left|e_{j}\right\rangle=\sum_{m} U_{3 n}^{-1}|m\rangle \\
& \left|f_{k}\right\rangle=\sum_{n=1}^{N-1} \sum_{n=n-1}^{N} v_{n m}^{i k 1}|m . n\rangle .
\end{aligned}
$$

where $|m\rangle=a_{m \mid}^{-}|0\rangle$ and $|m, n\rangle=a_{m}^{-} a_{n}^{-}|0\rangle$. The eigenvector elements of the $f^{\text {th }}$ one-exciton and the $k^{\text {th }}$ two-exciton states were denoted as $U_{3 m}^{-1}$ and $v_{m n}^{i(k)}$, respectively. The matrix elements of $v^{(k)}$ correspond to the elements of the $k^{\text {th }}$ row of the matrix $V^{-1}$.

Due to the chromophore-bath interaction, the corresponding matrices $H_{S B}^{(1)}$ and $H_{S B}^{(2)}$ in the site representation can be transformed as. in the delocalized exciton state representation,

$$
\begin{aligned}
& \hbar \tilde{\Xi}_{S B}^{(1)}(\mathbf{Q})=U^{-1} \tilde{H}_{S B}^{\prime l)}(\mathbf{Q}) U \\
& \hbar \tilde{\Xi}_{S B}^{(\hat{-1}}(\mathbf{Q})=V^{-1} \tilde{H}_{S B}^{\hat{-1}}(\mathbf{Q}) V .
\end{aligned}
$$

The diagonal matrix elements. $\left[\tilde{\Xi}_{S B}^{i l]}(\mathbf{Q})\right]_{j j}$ and $\left[\tilde{\Xi}_{S B}^{(2)}(\mathbf{Q})\right]_{k k}$, describe the energy fluctuations induced by chromophorebath interaction of the $f^{\text {th }}$ one-exciton and the $k^{\text {th }}$ two-exciton states, respectively. The off-diagonal matrix elements of $\hat{\Xi}_{S B}^{(1)}(\mathbf{Q})$ and $\tilde{\Xi}_{S B}^{(2)}(\mathbf{Q})$ will induce exciton relaxations witlin the one-and two-exciton state manifolds. respectively.

C. Exciton state energy fluctuations. Now. the energy of the $f^{\text {th }}$ one-exciton state. fluctuating due to chromophorebath interactions. can be written as

$$
\hbar \Omega_{j l}(\mathbf{Q})=\hbar \tilde{\Theta}_{i j}+\hbar\left[\tilde{\Xi}_{S B}^{i 1)}(\mathbf{Q})\right]_{i j}=\hbar \tilde{\Omega}_{j i}+\hbar \sum_{m} \sum_{n} l_{j m}^{-1} \vartheta_{m n}(\mathbf{Q}) l_{m}
$$

Usually. the fluctuation amplitudes of the coupling constants. $q_{m+n}($ for $m \neq n)$, are smaller than the fluctuation amplitudes of site energies (diagonal elements), i.e.

$$
\left.\left\langle q_{n m}^{2}\right\rangle\right\rangle\left\langle\left\langle q_{n p}^{2}\right\rangle \text { for all } m . n \text {, and } p \text {, and } n \neq p\right. \text {. }
$$

Therefore. Eq. (22) can be simplified as

$$
\hbar \Omega_{j}(\mathbf{Q}) \equiv \hbar \tilde{\Omega}_{j, l}+\hbar \sum_{n i} \hat{U}_{m j}^{\prime} q_{m m}(\mathbf{Q})
$$

Note that the fluctuation of the $j^{\text {th }}$ one-exciton state energy, the second term in Eq. (24). is given by a linear combination of each site energy fluctuation term. $\hbar_{q_{m m}}(\mathbf{Q})$. and the weighting factors are determined by the square of the corresponding eigenvector elements. Similarly, the $k^{\text {th }}$ twoexciton state energy is written as 


$$
\begin{aligned}
& \hbar W_{k}(\mathbf{Q})=\hbar \tilde{W}_{k k}+\hbar\left[\tilde{\Xi}_{2}^{e-p}(\mathbf{Q})\right]_{k k} \\
& =\hbar \tilde{W}_{k k}+\hbar \sum_{m} \sum_{n} V_{j m}^{-1}\left[\tilde{H}_{2}^{e-p}(\mathbf{Q})\right]_{m n} V_{n j} \\
& \cong \hbar \tilde{W}_{k k}+\hbar \sum_{m=1}^{N-1} \sum_{n=m+1}^{N}\left(v_{m n}^{i k j}\right)^{2}\left\{q_{m n}(\mathbf{Q})+q_{n n}(\mathbf{Q})\right\} .
\end{aligned}
$$

Note that the energy fluctuation at the $m^{\text {th }}$ site. described by $\hbar q_{m m}(\mathbf{Q})$. modulates both the one- and two-exciton state energies and the relative weighting factors are determined by the associated eigenvector matrix elements. From Eqs. (24) and (25), one can deduce the fact that the fluctuation of the $f^{\text {th }}$ one-exciton transition frequency is intrinsically correlated with the fluctuation of other one- or two-exciton transition frequencies. Because of this instantaneous correlation mediated by the electronic couplings. the one- and two-color photon echo peak shifts ${ }^{31}$ can be used to study the spatial extent of exciton delocalization as well as the spatial overlap between different one or two-exciton state probability densities.

D. Transition dipoles. Once the eigenvectors of the oneand two-exciton states are determined. the exciton transition dipole matrix elements can be expressed as linear combinations of transition dipoles of each chromophore i.e.

$$
\begin{aligned}
& \mu_{e_{j}} \equiv\left\langle 0|\hat{\mu}| e_{j}\right\rangle=\sum_{m} U_{j m}^{-1} d_{m} \\
& \mu_{e_{j} t_{k}} \equiv\left\langle e_{j}|\hat{\mu}| f_{k}\right\rangle=\sum_{m=1}^{N-1} \sum_{n=m+1}^{N} v_{m m}^{(k j)}\left(U_{m n}^{-1} d_{m}+U_{j m}^{-1} d_{n}\right)
\end{aligned}
$$

where $d_{m}$ is the transition dipole vector of the $m^{\text {th }}$ cluromophore, i.e. $d_{m} \equiv\langle 0|\hat{\mu}| m\rangle$. where $\hat{\mu}$ is the electric dipole operator. These transition dipole matrix elements in Eq. (26) are used to calculate various 2D spectroscopic response functions of coupled multi-chromophore systems. such as polypeptides and light-harvesting complexes.

E. Frequency-frequency correlation functions. For a multi-level system. we need both the auto- and crosscorrelation functions of the one- and two-exciton transition frequencies to eventually calculate the 2D spectroscopic nonlinear response functions. Using the approximate expressions in Eq. (24). one can obtain the time-correlation between any given two one-exciton transition frequencies as

$$
\left\langle\delta \Omega_{j}(t) \delta \Omega_{k}(0)\right\rangle=\sum_{m} \sum_{n} U_{m j}^{2} U_{n k}^{2}\left\langle q_{m m}(t) q_{n m}(0)\right\rangle
$$

where $\quad \delta \Omega_{j}(\mathbf{Q})=\Omega_{j}(\mathbf{Q})-\tilde{\Omega}_{j j} \quad$ and $\quad \delta \Omega_{j}(t)=\exp \left(i H_{p h} t / \hbar\right)$ $\delta \Omega_{j}(\mathbf{Q}) \exp \left(-i H_{p h} t / \hbar\right)$. If the energy fluctuation at the $m^{\text {th }}$ chromophore is statistically independent of the energy fluctuation at the $n^{\text {th }}$ chromophore, i.e.,

$$
\left\langle q_{m m}(t) q_{m n}(0)\right\rangle=\delta_{m n}\left\langle q_{m m}(t) q_{m m}(0)\right\rangle .
$$

then. Eq. (27) simplifies to

$$
\left\langle\otimes \Omega_{j}(t) \& \Omega_{k}(0)\right\rangle=\sum_{m} U_{m j}^{2} U_{n k}^{2}\left\langle q_{m m s}(t) q_{m m s}(0)\right\rangle .
$$

We further assume that the site energy fluctuation correla- tion functions $\left\langle q_{m m}(t) q_{m m t}(0)\right\rangle$ are all identical so that we have

$$
\left\langle q_{m m}(t) q_{n m i}(0)\right\rangle=C(t) \quad(\text { for all } m) .
$$

In order to express $C(t)$ in terms of spectral density. $C(t)$ is expressed as a sum of the real and imaginary parts as

$$
C(t)=a(t)+i b(t) .
$$

Introducing the spectral density $\rho(\omega)$ representing the spectral distribution of the chromophore-bath coupling constants, ${ }^{24}$ one can rewrite the real and imaginary parts. $a(t)$ and $b(t)$, as

$$
\begin{aligned}
& a(t)=\int_{0}^{\infty} d \omega \rho(\omega) \operatorname{coth} \frac{\hbar \omega}{2 k_{B} T} \omega^{2} \cos \omega t . \\
& b(t)=\int_{0}^{\infty} d \omega \rho(\omega) \omega^{2} \sin \omega t .
\end{aligned}
$$

Note that the absolute magnitude of the spectral density is determined by the solvent reorganization energy as $\lambda=$ $\hbar \int_{0}^{\infty} d \omega \omega \rho(\omega),{ }^{2-4}$ and $\lambda$ is one-quantum energy averaged over the spectral density. Since the initial value of $C(t)$. which is the mean square fluctuation amplitude of the transition frequency of an isolated chromophore. is a critical quantity. it is especially denoted as

$$
\begin{aligned}
C_{0} & =C(0)=\left\langle\omega^{2} \operatorname{coth}\left[\hbar \omega / 2 k_{B} T\right]\right\rangle_{\rho} \\
& =\int_{i j}^{\infty} d \omega \rho(\omega) \operatorname{coth}\left[\hbar \omega / 2 k_{B} T\right] \omega^{2} .
\end{aligned}
$$

By using the approximation of Eq. (30), Eq. (29) is further simplified as

$$
\otimes_{j}(t) \otimes_{h}(0)=\left(\sum_{m} U_{m j}^{2} U_{m i k}^{2}\right) C(t)
$$

We next consider the correlation functions between any given two two-exciton transition frequencies and they are found to be

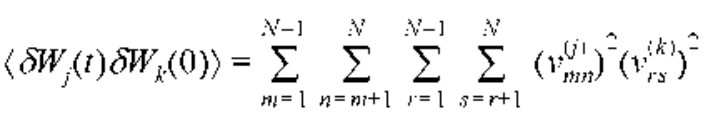

$$
\begin{aligned}
& \times\left\langle\left\{q_{m m}(t)+q_{m}(t)\right\}\left\{q_{r r}(0)+q_{s s}(0)\right\}\right\rangle \\
& \equiv\left(\sum_{m=1}^{N-1} \sum_{n=m-1}^{N}\left(v_{n m}^{(j)}\right)^{2}\left\{P_{n i}^{(k)}+P_{n}^{i k \mid}\right\}\right) C(t)
\end{aligned}
$$

where the second equality was obtained by invoking the approximation in Eq. (30), and

$$
P_{m}^{(k)} \equiv \sum_{j=1}^{m-1}\left(v_{j m}^{(k)}\right)^{2}+\sum_{j=m+1}^{N}\left(v_{m j}^{i k}\right)^{2} .
$$

In addition to the correlation functions between two oneexciton transition frequencies and between two two-exciton transition frequencies. the cross-correlation functions between $\delta \delta_{j}(t)$ and $\delta W_{k}(0)$ are required in calculating the nonlinear response functions. They are 


$$
\begin{aligned}
& \left\langle\delta \Omega_{j}(t) \delta W_{k}(0)\right\rangle \\
& \quad=\sum_{m=1}^{N} \sum_{r=1}^{N-1} \sum_{s=r+1}^{N} U_{m j}^{i}\left(v_{r s}^{\prime h^{\prime}}\right)^{2}\left\langle q_{m m}(t)\left\{q_{r r}(0)+q_{s s}(0)\right\}\right\rangle \\
& \cong\left(\sum_{m=1}^{N} U_{m j}^{2} P_{m}^{(k)}\right) C(t)
\end{aligned}
$$

In this subsection. involing the two approximations. Eqs. (28) and (30). we showed that all auto- and cross-correlation functions of fluctuating one- and two-exciton frequencies can be written in terms of the frequency-frequency correlation function of a single cluomophore, $C(t)$.

F. Delocalization and spatial overlap of excitons. Three different correlation functions of one- and two-exciton state frequency fluctuations have different physical meanings and are related to either the spatial delocalization of the corresponding exciton or the spatial overlap between the excitonic state probability densities. First of all. the mean square fluctuation amplitude of the $j^{\text {th }}$ one-exciton transition frequency, $\left\langle\delta \Omega_{j}^{2}\right\rangle$ is given as

$$
\frac{\left\langle\delta \Omega_{j}^{2}\right\rangle}{C_{p}}=\sum_{m} U_{m j}^{+}=\frac{1}{N_{j}} .
$$

Noting that the inverse participation ratio (IPR) of the $j^{\text {th }}$ one-exciton state $N_{j}$ is defined as $N_{j}=\left[\sum_{m} U_{m j}^{4}\right]^{-1}$, we find that the ratio $\left\langle\delta \Omega_{j}^{2}\right\rangle / C_{i}$ can be of use to obtain the extent of delocalization of the jth exciton state in space. As $\mathrm{Nj}$ increases. the associated line width. which is approximately proportional to $\left\langle\delta \Omega_{j}^{2}\right\rangle$. decreases. This is a well-known exchange-narrowing effect found in a $J$-aggregate ${ }^{32}$ In order to measure this auto-correlation function. $\left\langle\delta \Omega_{j}(T) \delta \Omega_{j}(0)\right\rangle$. one can use the photon echo peak shift measurement method. It was found that the photon echo peak shift decay's. with respect to $T$. as ${ }^{2.33}$

$$
\tau^{*}\left(T: \omega_{1}=\omega_{3}=\bar{\omega}_{e_{j} \beta}\right)=\frac{\operatorname{Re}\left[\left\langle\delta \Omega_{j}(T) \delta \Omega_{j}(0)\right\rangle\right]}{\sqrt{\pi}\left\langle\delta \Omega_{j}^{2}\right\rangle^{3: 2}} .
$$

where the center frequencies of the fentosecond laser beans. (i) and $\omega_{3}$. are tuned to be identical to the average transition frequency of the one-exciton $\mid e_{j}>$ state.

Secondly, the normalized cross correlation anplitude. $\left\langle\delta \Omega, \delta \Omega_{k}\right\rangle / C_{0}$, is written as

$$
\frac{\left\langle\delta \Omega_{j} \delta \Omega_{h}\right\rangle}{C_{0}}=\sum_{m} U_{m j}^{2} U_{m k}^{2} .
$$

The vector. $\vec{p}_{j}=\left(U_{1 j}^{2}, U_{z_{j}}^{2} \ldots\right)$. where each element is the square of eigenvector element. describes the probability density distribution of the $j^{\text {th }}$ exciton state in the site representation. Thus. we have

$$
\left\langle\infty \Omega_{j} \infty \Omega_{k}\right\rangle / C_{0}=\vec{p}_{j} \cdot \vec{p}_{k}
$$

which can be viewed as the spatial overtap of the two probability density distributions. $\vec{p}_{j}$ and $\vec{p}_{k}$, of the $j^{\text {th }}$ and $k^{\text {th }}$ exciton states. This suggests that $\left\langle\delta \Omega_{j} \delta \Omega_{h}\right\rangle / C_{i j}$ is a measure of overlap between $\left|\psi_{i}\right|^{2}$ and $\left|\psi_{k}\right|^{-}$. where $\psi_{3}$, for example, is the wavefunction of the $j$ th one-exciton state. Once $\left\langle\delta \Omega_{j} \delta \Omega_{k}\right\rangle / C_{i j}$ values are experimentally measured, it will provide spatial information about a pair of excitons that have different frequencies. Recently. Cho and Fleming showed that the two-color photon echo peak shift measurement. where the two different frequencies of the incident laser beams are simultaneously resonant with the $j^{\text {th }}$ and $k^{\text {th }}$ oneexciton states. can be used to measure this particular quantity: i.e. ${ }^{2-4}$

$$
\tau_{\text {rud }}^{*}\left(T: \omega_{1}=\bar{\omega}_{e_{, g} \cdot \omega_{3}}=\bar{\omega}_{e_{k g}}\right)=\frac{\operatorname{Re}\left[\left\langle\delta \Omega_{j}(T) \delta \Omega_{k}(0)\right\rangle\right]}{\sqrt{\pi}\left\langle\delta \Omega_{j}^{2}\right\rangle\left\langle\delta \Omega_{k}^{2}\right\rangle^{1 ; 2}} .
$$

Next. let us consider the cross-correlation between the $l^{\text {th }}$ one-exciton state frequency fluctuation and the $k^{\text {th }}$ twoexciton state frequency fluctuation. $\left\langle\delta \Omega_{j}(t) \delta W_{k}(0)\right\rangle$. We find that

$$
\frac{\left\langle\delta \Omega_{j} \delta W_{k}\right\rangle}{C_{0}}=\sum_{m=1}^{N} U_{m\}}^{2} P_{n i}^{i k !} .
$$

In order to understand the physical meaning of the quantity. $\sum_{m=1}^{N} V_{n j}^{2} P_{n j}^{k i}$. let us define the projection operator. $\hat{P}_{n \eta}=|m\rangle\langle m|$. where $|m\rangle$ denotes the singly excited state of the $m^{\text {th }}$ cluromophore. Then, one can prove that

$$
P_{m}^{(k)}=\left\langle f_{k}\left|\hat{P}_{m}\right| f_{k}\right\rangle
$$

where $\left|f_{k}\right\rangle$ is the $k^{\text {th }}$ two-exciton state wavefunction. i.e.,

$$
\left|f_{k}\right\rangle=\sum_{n=1}^{N-1} \sum_{n=n i-1}^{N} v_{m n}^{(k)}|m\rangle|n\rangle
$$

Therefore. $P_{m}^{(k)}$ is the expectation value of $\hat{P}_{m}$ over the probability distribution of the $k^{\text {th }}$ two-exciton state. and is considered to be the "amount" of the $\mid m>$ wavefunction in the $k^{\text {th }}$ two-exciton state. In other words. $\vec{P}_{k} \equiv\left(P_{1}^{(k)}, P_{2}^{(k)}, \ldots\right)$ vector is the reduced (projected) probability density of each site in the $k^{\text {th }}$ two-exciton state. Therefore. Eq. (44) can be rewritten as,

$$
\frac{\delta \Omega_{j} \delta W_{k}}{C_{\varphi}}=\vec{p}_{l} \cdot \vec{p}_{k}
$$

which can be interpreted as the spatial overlap between the probability density of the $f^{\text {th }}$ one-exciton and the reduced probability density of the $k^{\text {h }}$ two-exciton state.

Finally the mean square fluctuation amplitude of the $k^{\text {th }}$ two-exciton state is found to be

$$
\frac{\left\langle\delta W_{k}^{\hat{\rangle}}\right\rangle}{C_{0}}=\sum_{m=1}^{N-1} \sum_{n=m-1}^{N}\left(\left(_{n m}^{(k)}\right)^{\prime}\left\{P_{m}^{(k)}+P_{n}^{(k)}\right\}\right. \text {. }
$$

This result suggests that $\left\langle\delta \hat{W_{k}^{-}}\right\rangle$is determined by the overlap of the probability density of the $k^{\text {th }}$ two-exciton in the $|m . n\rangle$ basis and the projected (reduced) probability densities.

Now, we have described the nature of one- and twoexciton states. $\left|e_{j}\right\rangle$ and $\left|f_{k}\right\rangle$, and also presented the expressions for transition dipole matrix elements. Then, using the theoretical formula for 2D spectroscopic nonlinear 
response functions given in Sec.III and carrying out 2D Fourier transformation of the relevant nonlinear response function. one can calculate the 2D vibrational or electronic spectrum of any coupled multi-chromophore sy stem.

\section{Two-dimensional Vibrational Spectroscopy of Polypeptides}

In the case of 2D vibrational spectroscopy due to the doubly'-vibrationally-resonant condition. two different IR fields. for example. can be simultaneously in resonance with two different vibrational transitions. Furthermore, in order for the signal not to be zero. the vibrational coupling between two modes via mechanical or electric anhamonicities should not vanish. This is possible when the two vibrational chromophores interact with each other via through-bond or through-space interaction. Therefore. the 2D vibrational spectroscopy can be a useful method to examine whether the two vibrational chromophores are sufficiently close to each other. ${ }^{3(j)}$ This is how $2 \mathrm{D}$ vibrational spectroscopy can provide much more detailed information on three-dimensional molecular structure.

Similar to the 2D vibrational spectroscopy the 3D vibrational spectroscopy when the three different vibrational degrees of freedom are excited by the three beams with different frequencies can provide through-bond and/or through-space interaction network among the three modes (chromophores). This method therefore is a useful tool for measuring three-bod' interaction ${ }^{-10}$ and analogous to multidimensional hetero-nuclear NMR spectroscopy.

However. in order for 2D vibrational spectroscopic techuiques to be of use in probing structures and dynamics of proteins in solution. there are a number of issues to be addressed as summarized in Figure 6. For a given target vibrational degree of freedom. the solvation can induce both solvatochromic frequency shift and time-dependent frequency fluctuation leading spectral line broadening. For example. the hy'drogen-bonding effects on vibrational properties are to be understood for quantitatively establishing protein structure-spectra relationships in general. In addition to the solute-solvent interactions. the intramolecular vibrational interactions between different local sibrational modes should also be understood. For instance. the amide I mode localized at a given peptide bond in a protein backbone can interact with a neighboring peptide vibration. which also induces frequency shift and fluctuation as the protein structure constantly changes in time.

There exist quite a number of spectroscopic methods that have been used to probe structure and dynamics of biomolecules (see Fig. 6). The conventional ID IR absorption and Raman scattering methods have been served as the most vital tools for such purposes. Also a few vibrational optical activity measurement methods such as vibrational circular dichroism and Raman optical activity spectroscopies were found to be quite useful and infomative. In addition to the 2D vibrational (IR) spectroscopy utilizing either IR photon echo or IR pump-probe scheme. it was theoretically pro-
Probing Structure and Dynamics of Biomolecules

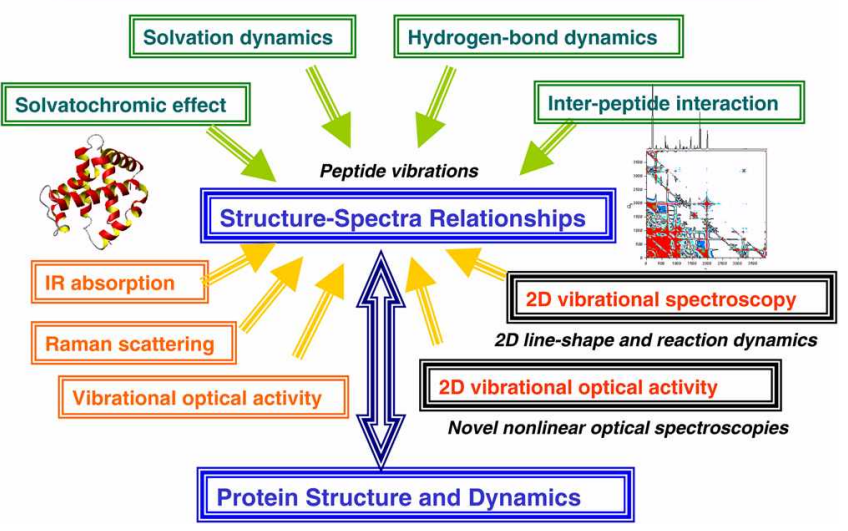

Figure 6. A schematic diagram showing issues to be studied and methods to be used to establish the structure-spectrum relationships.

posed that 2D vibrational optical activity spectroscopy utilizing circularly polarized beams would provide far detailed information on structure of chiral molecules such as proteins and other biomolecules. ${ }^{2}$

In this chapter. we will present brief reviews on the theoretical studies of vibrational dynamics of proteins. from a single peptide molecule to various secondary structure polypeptides in solution.

A. N-Methylacetamide: a prototype peptide model system. Hydration effects on the molecular structure and amide I mode frequency of a prototype peptide molecule. $N$ methylacetamide (NMA), when it is solvated by a few water molecules. were investigated by carrying out $a b$ initio calculations for a number of randomly chosen NMA-water clusters (see Fig. 7 for example). ${ }^{35}$ The linear relationship between the structural distortion of peptide bond. i.e.. $\mathrm{C}=\mathrm{O}$ bond length. and the amide I mode frequency was described by using the theory presented in Ref. [36]. The harmonic frequency shift of the amide I mode in NMA-n $\mathrm{D}_{2} \mathrm{O}(n=1-5)$ complex was found to originate from the combination of the molecular cubic anhamonicity and displacement of the amide I coordinate when the NMA is hydrated. ${ }^{35}$ Using a multivariate least square fitting method, the effective transition charges of six NMA sites were detemined. Then, this empirical model was successfully used to quantitatively describe solvatochromic frequency shift of the NMA amide I

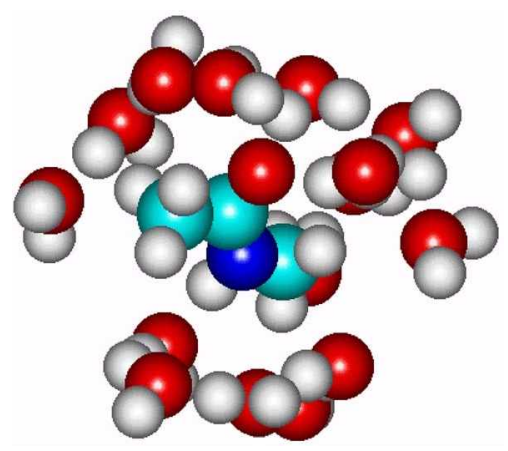

Figure 7. An NMA-water cluster. 


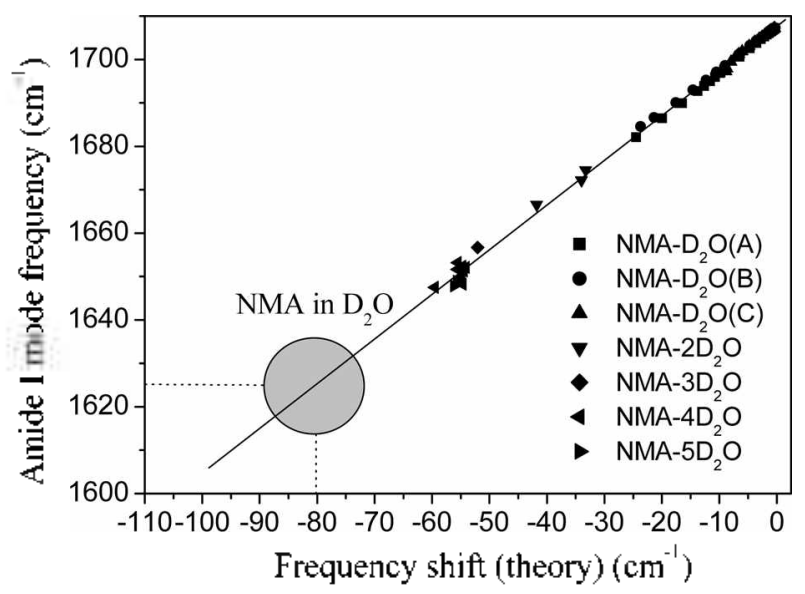

Figure 8. Amide I mode frequencies of NMA-water clusters are on the $\gamma$-axis. The multivariate least square fitting results are on the $\mathrm{x}$ axis, and the correlation appears to quantitative (see Ref. [35] for a more detailed description).

mode upon hydration.

Once the amide I mode frequency shift of NMA in NMAwater clusters is quantitatively detemined. one can use the theoretical model to describe amide I mode frequency shift and fluctuation of NMA in water in combination with MD simulations of an NMA in $\mathrm{H}_{-} \mathrm{O}$ and $\mathrm{D}_{2} \mathrm{O}$ solutions. The ensemble averaged amide I mode frequency shift was found to be $-78 \mathrm{~cm}^{-1}$ in comparison to that of the gas-phase NMA molecule. which was found to be in excellent agreement with the experimental value of $-81 \mathrm{~cm}^{-1}$ (Fig. 8). ${ }^{37}$ Sumilar to the solvation correlation function of a polar solute in water the amide I mode frequency-frequency correlation function exhibits a bimodal decaying pattern and both the hindered translational and the librational motions of the water molecules directly hydrogen-bonded to the NMA were found to play critical roles in the pure dephasing of the amide I mode ${ }^{3 \mathrm{~S}}$ The pure dephasing constant was estimated to be $11 \mathrm{~cm}^{-1}$. It was shown that the vibrational broadening mechanism is mainly determined by the motional narrowing process. The vibrational Stokes shift of the amide I mode was estimated to be as small as $1.2 \mathrm{~cm}^{-1}$. The anide I IR absorption spectrum thus calculated without any adjustable parameters except for the lifetime of the first excited state has a full width at half maximum of $26.9 \mathrm{~cm}^{-1}$ and is found to be in good agreenent $w$ ith the experinent. ${ }^{39-41}$

Next, we used this method to numerically simulate 2D IR pump-probe spectrum of NMA in liquid water. which was experimentally studied by Woutersen et $a^{39}$ and Zanni et $a l^{21 j}$ We presented a theoretical description of two-dimensional (2D) IR pump-probe spectroscopy of a three-level system by taking into account the system-bath interaction properly. ${ }^{2}$ Note that there are six different nonlinear optical transition pathways depicted in Figure 9. where the ground. first excited. and second excited vibrational states are denoted as $|0\rangle, \mid 1>$, and $|2\rangle$. respectively. The incoming and outgoing (short) arrows represent the absorptive and emisive field-matter interactions. respectively. The transient (excited state) absorption contribution involves a transition from the
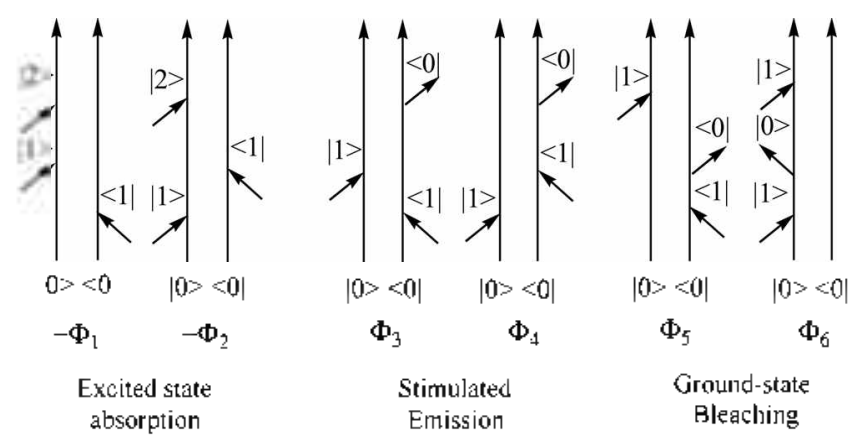

Figure 9. Six double-sided Feynman diagrams associated with the 2L) IR pump-probe spectroscopy. The vibrational ground, first excited, and second excited states are denoted as $|0\rangle, 1\rangle$, and $|2\rangle$. respectively. The first two pathways are excited state absorption (EA) and contribute to the pump-probe signal negatively due to the absorptive field-matter interaction. The third and fourth diagrams are stimulated emission (SE) terms, and the fifth and sixth are ground-state bleaching (GB). Note that the SE and GB pathways involve transitions between the ground and first excited states. Adapted from [42]

first excited state to the second excited state so that its contribution to the pump-probe signal is negative. On the other hand the ground-state bleaching and stimulated emission contributions to the signal are positive. Thus. the quantum interference at the amplitude level is critical in this nonlinear vibrational spectroscopy and the lack of perfect destructive interference due to finite vibrational anharmonicities is the key for interpreting 2D IR pump-probe signals from an anhannonic oscillator system such as the amide I vibration of NMA molecule.

By using the correlation function of the fluctuating amide I mode frequency of NMA in $\mathrm{D}_{2} \mathrm{O}$, which was obtained by combining both $a b$ initio calculations with $\mathrm{MD}$ simulation results. the time-resolved 2D pump-probe spectra as a function of pump-probe pulse delay time $(T)$ were calculated and are plotted in Figure 10.

It was found that the vibrational dephasing becomes homogeneous in $2 \mathrm{ps}$ time scale. which is a bit faster than the experimental result ( $\sim \mathrm{ps}$ ). We also showed that the degree of slant of $2 \mathrm{D}$ contours. $\sigma_{F P}(T)$. is inversely proportional to the correlation function of the fluctuating amide I mode frequency. i.e. $1 / \sigma_{P P}(T)=\langle\delta \omega(T) \delta \omega(0)\rangle /\langle\delta \omega\rangle$, where $\sigma_{F P}(T)$ is the slope of the tangential line shown in the first panel of Figure 10 and $\delta(x)$ is the fluctuating amide I mode frequency. ${ }^{8}$ Consequently, we showed that the 2D IR pumpprobe spectroscopy can provide a direct information on the vibrational frequency fluctuation dynamics reflecting timedependent change of peptide-water interaction in time and on the magnitude of dynamic inhomogeneity. Although the 2D vibrational spectra shown in Figure 10 are specifically 2D IR pump-probe ones. one can also use the same argument to interpret the $2 \mathrm{D}$ line shape change of the 2D IR photon echo spectra with respect to the waiting time $T$.

Although the carbonyl oxygen atom of the NMA in water was found to maximally form $\mathrm{H}$-bonds with surrounding water molecules. that in liquid methanol can have two 


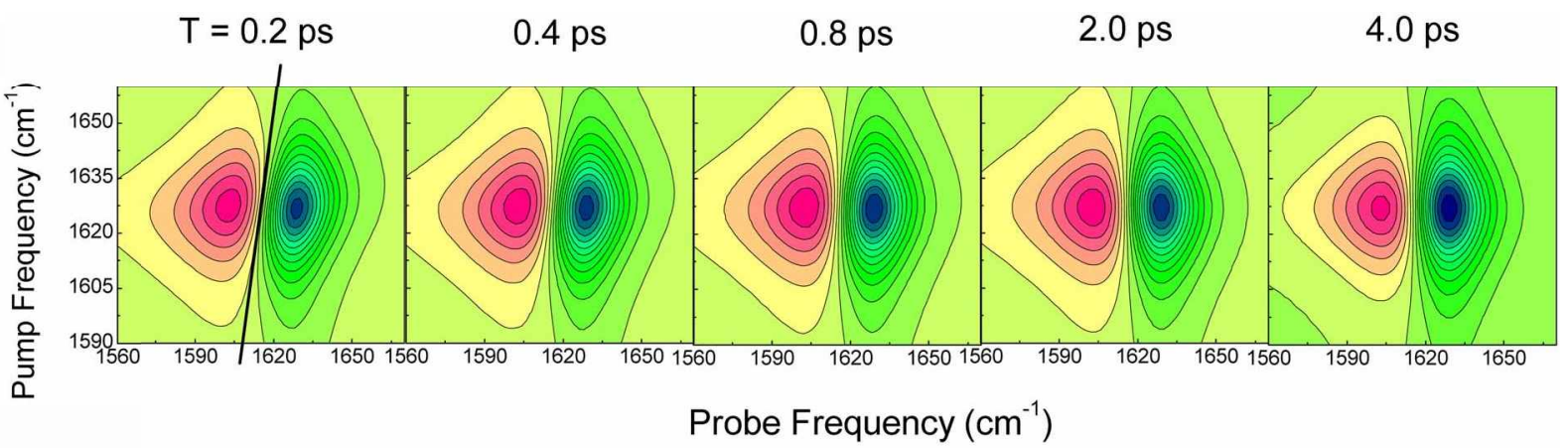

Figure 10. Time-resolved 2D IR pump-probe spectra of NMA in water. The waiting time $T$ vanes from 0.2 to $4.0 \mathrm{ps}$. Adapted from [42].<smiles>CC(=O)N(C)PCO[Mg]</smiles>
H-bond association<smiles>C#CC#N</smiles>

\section{$\mathrm{H}$-bond dissociation}

Figure 11. NMA molecule in methanol can have two different solvation structures. The two difter from each other by the number of hydrogen-bonded methanol molecules to the NMA carbonvl group. These two species are in thermal equilibrium and they can be separately identified in the IR absorption spectrum. The rate constants of hydrogen-bond association and dissociation processes can be estimated by measuring the time-dependent cross peak amplitudes in the 2D IR spectrum.

different solvation structures differing from each other by the number of H-bonded methanol molecules (see Fig. 11). All other methanol molecules surround these two species in solution. Due to the presence of these two different solvation structures in MeOD. the amide I IR band exhibits a doublet feature. ${ }^{911}$ From the MD simulations. it was found that the low-frequency anide I band is associated with the amide I mode of NMA with two H-bonded methanol molecules at the carbonyl oxygen site (the structure on the right-hand side of Fig. 11), whereas the high-frequency anide I band is with that having one $\mathrm{H}$-bonded methanol molecule (that on the left-hand side of Fig. 11). Using an empirical correction method and carrying out $a b$ initio calculation studies of NMA- $\left(\mathrm{CH}_{3} \mathrm{OH}\right)_{n}$ clusters and MD simulations, ${ }^{11}$ we were able to quantitatively describe the solvatochromic vibrational frequency shift induced by the hydrogen-bonding interaction between NMA and solvent methanol. The amide I mode frequency distribution was found to be notably nonGaussian and it could be decomposed into two Gaussian bands that are associated with two distinctively different solvation structures (Fig. 11). The ensemble-averaged linear response function. which represents the relaxation of the amide I transition dipole-dipole correlation function. is found to be oscillating. which is the origin of the doublet amide I band in frequency domain. Numerically calculated infrared absorption spectrum was directly compared with experiment and the agreement was found to be quantitative.
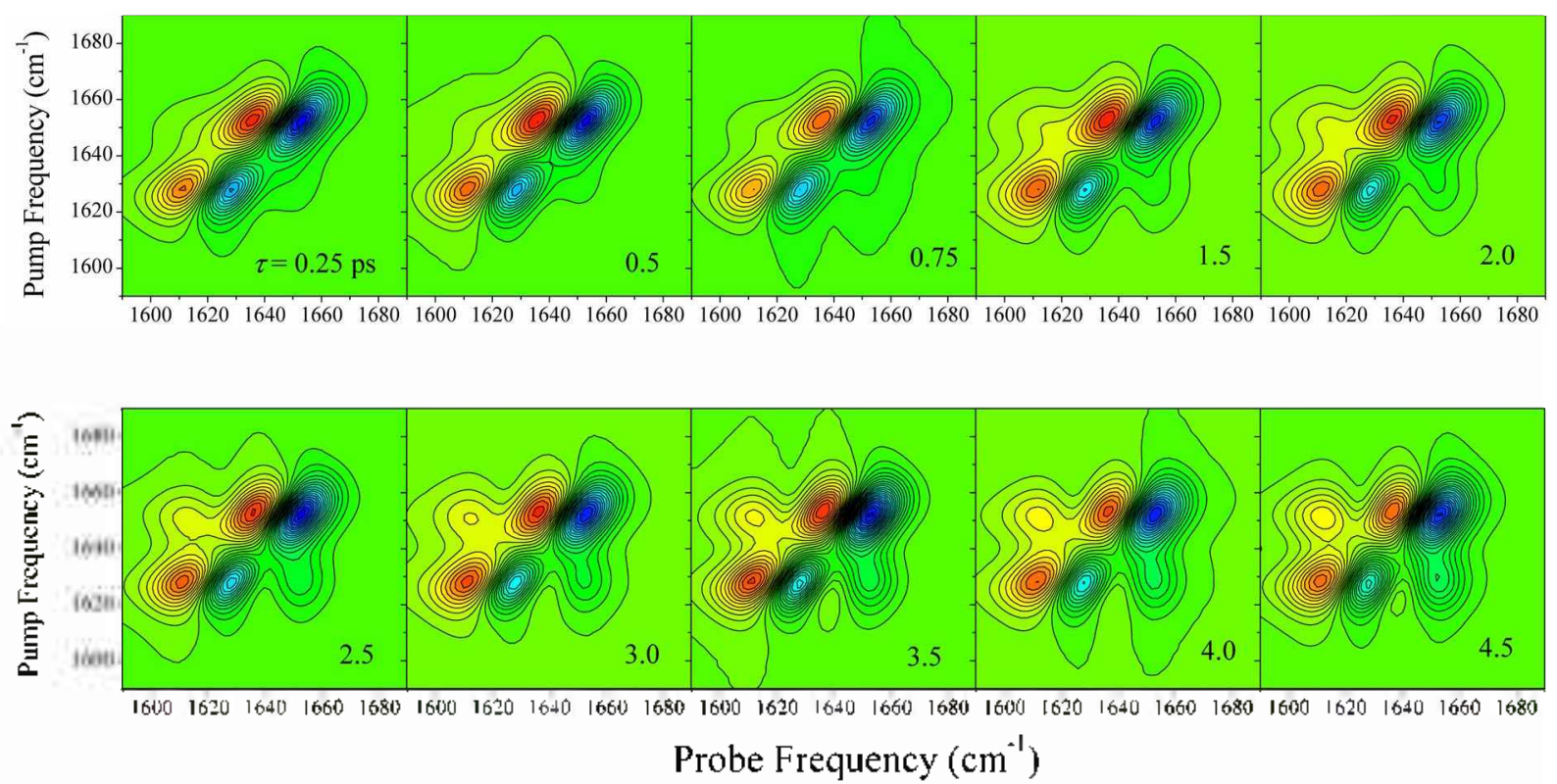

Figure 12. Time-resolved 2D IR pump-probe spectra of NMA in methanol. The waiting time varies trom 0.25 to 4.5 ps. Adapted trom [10]. 
By using the Onsager's regression hypothesis. the rate constants of inter-conversion process between the two solvation structures in Figure 11 were obtained. Then the nonlinear response functions associated with the $2 \mathrm{D}$ IR pump-probe spectroscopy were simulated and successfully compared with experimental result by Woutersen et al $^{9}$ and DeCamp et th $^{+1}$

As can be seen in Figure 12, as the waiting (population evolution) time increases from 0.25 to $4.5 \mathrm{ps}$. the cross peak amplitudes at $\left(\omega_{p n}=1630 \mathrm{~cm}^{-1} . \omega_{p r}=1650 \mathrm{~cm}^{-1}\right)$ and $\left(\omega_{p n}=\right.$ $\left.1650 \mathrm{~cm}^{-1}, \omega_{p r}=1650 \mathrm{~cm}^{-1}\right)$ increase. As theoretically described in detail in Refs. [10. 11]. this time-dependent cross peak amplitude changes are directly related to the $\mathrm{H}$ bond formation and dissociation dynamics and they can be quantitatively described by the conditional probability functions obtained by solving the relevant kinetic equations.

B. Dipeptide: $N$-acetylproline amide. Structure-spectrum relationship of a dipeptide that is the simplest model for polypeptides has been extensively studied by using a variety of spectroscopic methods such as IR absorption ${ }^{+3 .+4}$ vibrational circular dichroism. ${ }^{+5}$ non-resonant and resonant Raman scattering ${ }^{t i}$ and $2 \mathrm{D}$ IR spectroscopies. Among various polypeptide vibrations. the amide I band has been known to be highly sensitive to the secondary structure of polypeptide. There are a number of literatures and works that aim at determining solution structure of small peptides such as alanine dipeptide acetylproline amide (AP). etc.. These experimental studies utilizing IR. Raman. VCD , CD. and NMR methods show that the solution structure of these dipeptides are not random coil but close to the left-handed threefold helical polyproline II ( $\left.\mathrm{P}_{\mathrm{II}}\right)$ structure. Han et $\mathrm{al}^{47}$ carried out quantum chenistry calculation studies of alanine dipeptide with a few solvated water molecules and showed that the water bridge connecting the two carbonyl group in the dipeptide plays a critical role in stabilizing the dipeptide in its $\mathrm{P}_{11}$ structure. However, some experimental studies suggest that such solvent-mediated H-bonding interaction may' not be the determining factor for stabilizing $\mathrm{P}_{\mathrm{U}}-\mathrm{form}$ dipeptide in water but rather the intramolecular steric repulsive interaction plays the role. ${ }^{48}$

To elucidate the essential role of $\mathrm{H}$-bond network forming solvents. we studied the $\mathrm{N}$-acetylproline amide by using a few different spectroscopic methods. Before we discuss these works. it should be mentioned that Hochstrasser and coworkers have extensively studied the same dipeptide (AP) dissolved in either water or chloroform. employing the timeresolved 2D IR photon echo spectroscopic method. ${ }^{\text {[15.3] }}$ They showed that the vibrational coupling constant measured with such 2D IR method can be a good constraint that is of use to determine the solution structure of AP.

The AP (see Fig. 13 for its structure and notation of the two dihedral angles) contains two peptide bonds and their amide I local mode frequencies are separated from each other by about $30 \mathrm{~cm}^{-1}$ so that the amide 1 modes are relatively localized on one of the two peptide groups. ${ }^{52}$ Nevertheless. the two anharmonic anide I local modes are coupled to each other and they form two slightly delocalized

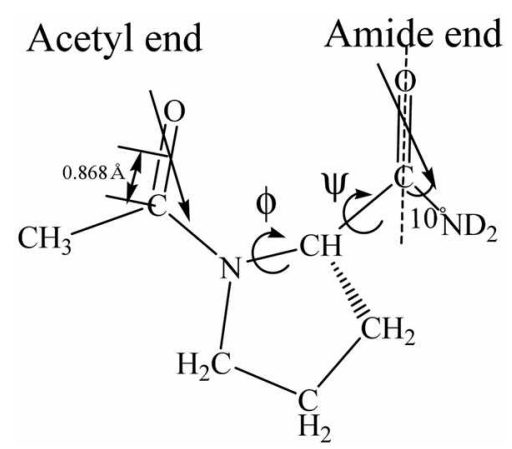

Figure 13. Molecular structure of $\mathrm{N}$-acetylproline amide. The two amide I local mode transition dipole vectors are shown in this figure as arrows. The two dihedral angles $\phi$ and $\psi$ detenmine the backbone structure of this dipeptide.

amide I normal modes. Therefore, cross peaks revealing the existence of coupling were experimentally observed. In the case of the aqueous AP solution. the two peptide groups form hydrogen bonds with water molecules in the first solvation shell. On the other hand. the chlorofonn solvent molecule cannot make a direct hydrogen bond with the AP peptide groups and thus the two peptide groups tend to form a direct intramolecular hydrogen bond to make its $C_{\text {Teq }}$ structure to be the global energy minimum conformation in a non-polar aprotic solvent. Therefore. the three-dimensional confomation of AP strongly depends on solvent. particularly on the hydrogen-bonding ability of the solvent molecules. Zanni et $\mathrm{dl}^{+9}$ were able to determine the absolute 3D confomations of AP in liquid water as well as chloroform by separately measuring the cross peak intensities of both parallel- and perpendicular-polarization photon echo signals.

In order to study the ensemble averaged structure of AP in solutions independently from the above mentioned 2D IR spectroscopic studies. we have carried out both experimental and theoretical studies. In order to detemine its aqueous solution structure. IR and VCD spectra of both L- and Dform AP solutions were measured. MD simulations with two different force fields $(f f 99$ and $f f 03$ parameters in AMBER7 and AMBER8 progranss, respectively') and density functional theory calculations for trans- and cis-rotamers of AP were performed to numerically simulate those spectra. ${ }^{53}$

Comparisons between experimentally measured and com-
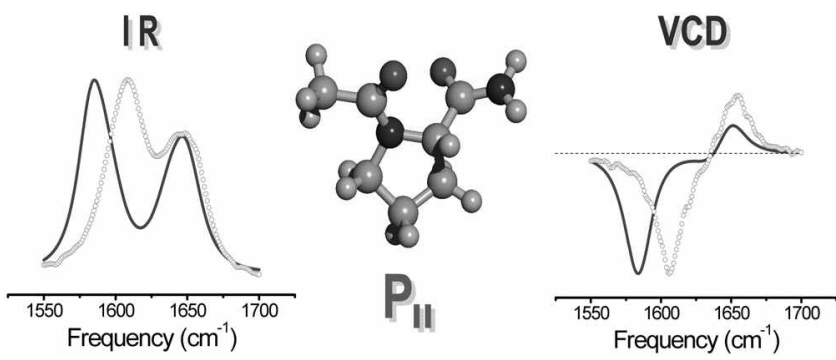

Figure 14. The $\mathrm{N}$-acetylproline amide adopts polyproline II structure in water. Esperimentally measured IR and V(CD) spectra (open circles) are directly compared with simulated ones (solid curves !. 


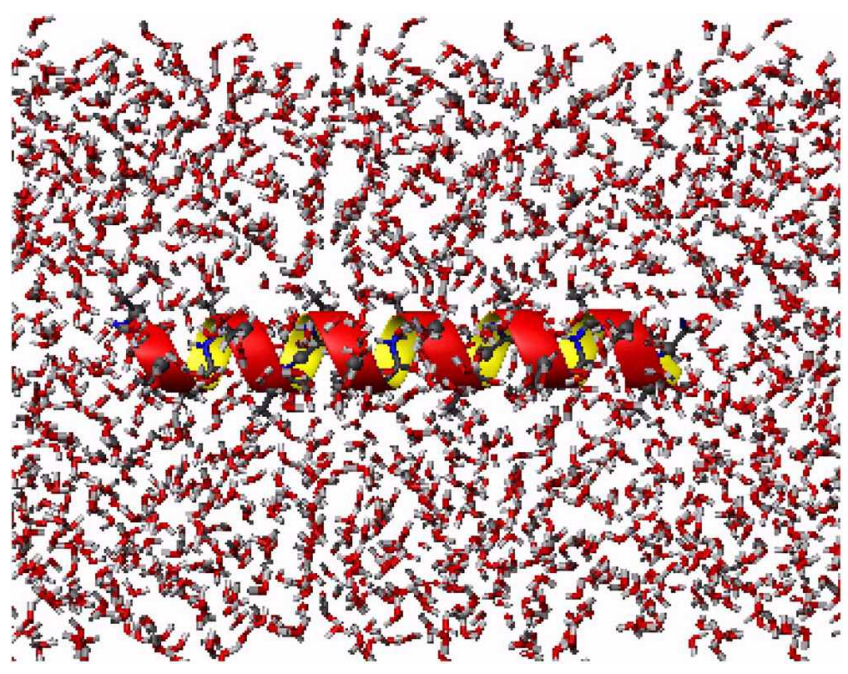

Figure 15. $\alpha$-helical polyalanine is put into TIP3P water molecules for molecular dynamics simulation studv.

putationally sinulated spectra directly suggest that the AP in water adopts a polyproline II-like conformation (see Fig. 14) and that the force field parameter $f f 03$ in AMBER8 suite of program is more realistic and reliable in predicting molecular structure of $\mathrm{AP}$ in water than the $f 99 \mathrm{in}$ AMBER7

C. $\alpha$-Helix. Chain length- and site-dependencies of amide I local mode frequencies of $\alpha$-helical polyalanines were theoretically studied by using our theoretical model that can be used to quantitatively predict both the local amide I mode frequencies and coupling constants between two different local amide I modes. ${ }^{54}$ Using this theoretical model and performing MD simulation of an $\alpha$-helical polyalanine in liquid water (see Fig. 15). we investigated conformational fluctuation and hydrogen-bonding dynamics of the $\alpha$-helix in water by monitoring the amide I frequency fluctuations and by analyzing the line shapes of the polarization-controlled 2D spectra.

The instantaneous nomal mode analysis method was first used to obtain densities of states of the amide I one- and twoexciton bands and to examine the extent of delocalization of the instantaneous amide I normal modes. Also. by introducing a novel concept of the so-called weighted phasecorrelation factor. the sy'mmetric natures of the delocalized amide I nomal modes were elucidated and also it was shown that there is no unique and simple way to classify the amide I nomal modes of the $\alpha$-helical polyalanine in water to be either $A$-mode-like or $E_{1}$-mode-like. From the ensemble-averaged dipole strength spectrun and density of oneexciton states. the amide I IR absorption spectrum was numerically calculated and its asymmetric line shape was theoretically described. Considering both transitions from the ground state to one-exciton states and those from oneexciton states to two-exciton states. we calculated the 2D IR pump-probe spectra (see Fig. 16) and directly compared them with experimental results presented by Hamm and coworkers

Amide I IR, VCD, and 2D IR spectra of various isotopelabeled $\alpha$-helical polyalanines in water were theoretically

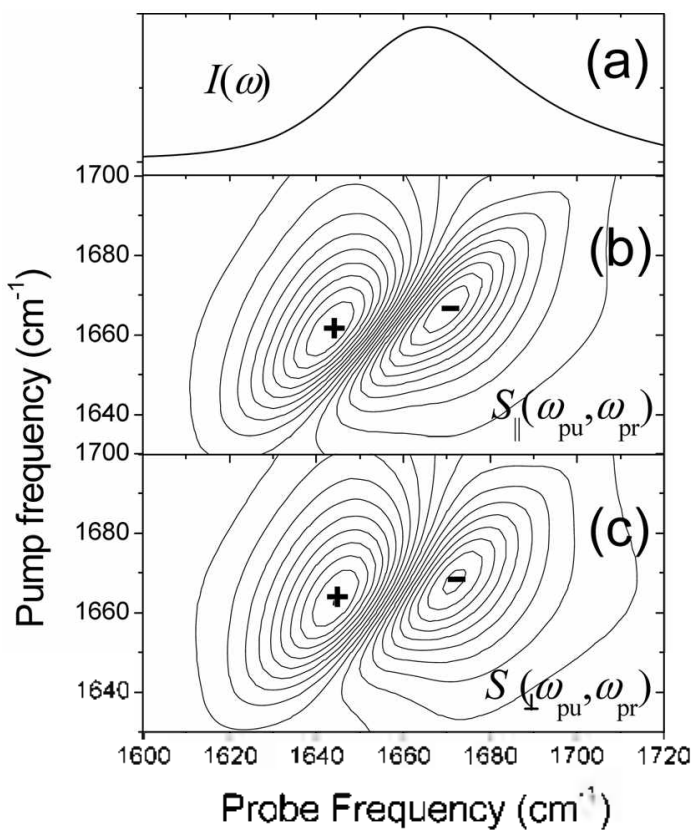

Figure 16. (a) Theoretically simulated amide I IR absorption band of $\alpha$-helis in water. 2D IR pump-probe spectra $S_{(}\left(\alpha_{p w}, \alpha_{p r}\right)$ and $S_{-}\left(\omega_{p r}, \omega_{p r}\right)$ are plotted in figures $(\mathrm{b})$ and $(\mathrm{c})$, respectively. Note that the polarization directions for $S\left\{\left(\alpha_{p t s}, \omega_{p r}\right\}\right.$ measurement are all parallel to laboratory $Z$-axis, where the beams propagate along the $Y$-axis. In the case of $S_{-}\left(\alpha_{m, n}, \alpha_{m}\right)$ measurement the pump beam polarization is perpendicular to the probe beam polarization. Adapted from [54]

calculated by combining semiempirical quantum chemistry calculation. Hessian matrix reconstruction, fragmentation approximation. and molecular dynamics simulation methods. ${ }^{56}$ The solvation-induced amide I frequency shift was found to be about $-20 \mathrm{~cm}^{-1}$. Properly taking into account the motional narrowing effect on the vibrational dephasing and line broadening processes. we showed that the simulated IR. VCD, and 2D IR spectra are quantitatively in good agreement with experimental results. Depending on the relative positions of the ${ }^{13} \mathrm{C}$ and $/$ or ${ }^{13} \mathrm{C}={ }^{18} \mathrm{O}$ labeled peptides in a given $a$-helix, the IR absorption line shape. IR intensity distribution. positive-negative VCD pattern. and diagonal/ off-diagonal 2D IR echo spectral features were found to change dramatically. It was shown that these different spectroscopic observations can be described in a consistent manner by using the present simulation method and coupled exciton model outlined in Sec.IV. Therefore. properly designed isotopomers and their IR. VCD. and 2D IR spectrum analyses can provide incisive information on the (local) three-dimensional polypeptide structure and dynamics.

D. $\beta$-Hairpin. The $\beta$-hairpin secondary structure motif has been extensively studied because its formation and dynamics are in many cases critical in understanding protein folding and unfolding dynamics. The instantaneous nonmal mode analysis of a $\beta$ hairpin in water was performed by using snapshot configurations sampled from the equilibrium MD trajectory. In order to fully take into account the motional and exchange narrowing processes and cross 

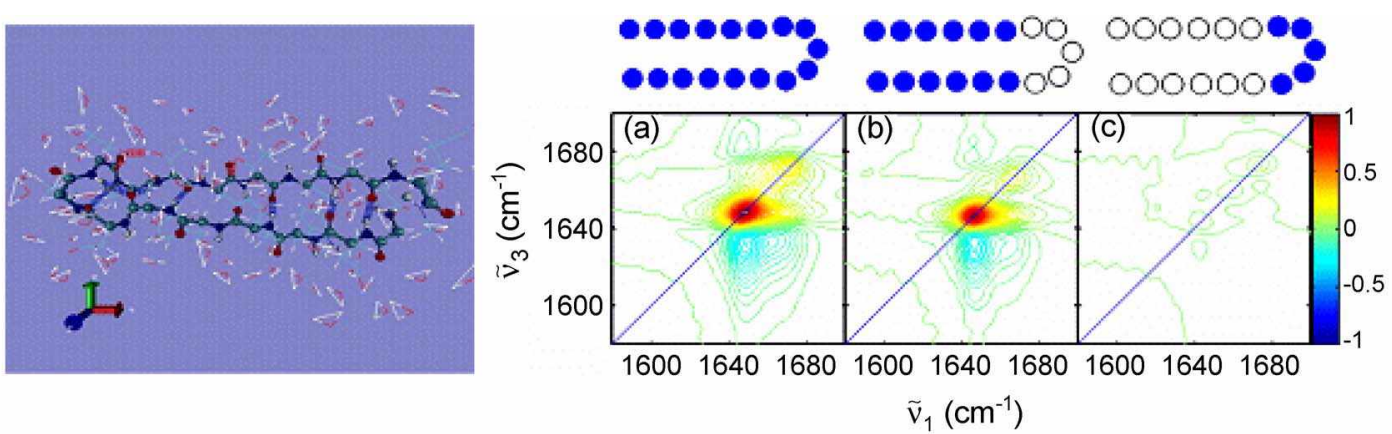

Figure 17. A snapshot picture of a $\beta$ hairpin in liquid water. The 2D IR photon echo spectrum of the model $\beta$ hlairpin is shown in figure (a) If those peptides in the two anti-parallel $\beta$-sheet strands (in the turn region) are only considered in the numerical simulation of $2 \mathrm{D}$ IR spectrum, the resultant spectra are shown in figures (b) ((c)). It turns out that the entire 2D spectrum (a) is largely dictated by the amide I modes delocalized on the two $\beta$-sheet branches. Adapted from [57]
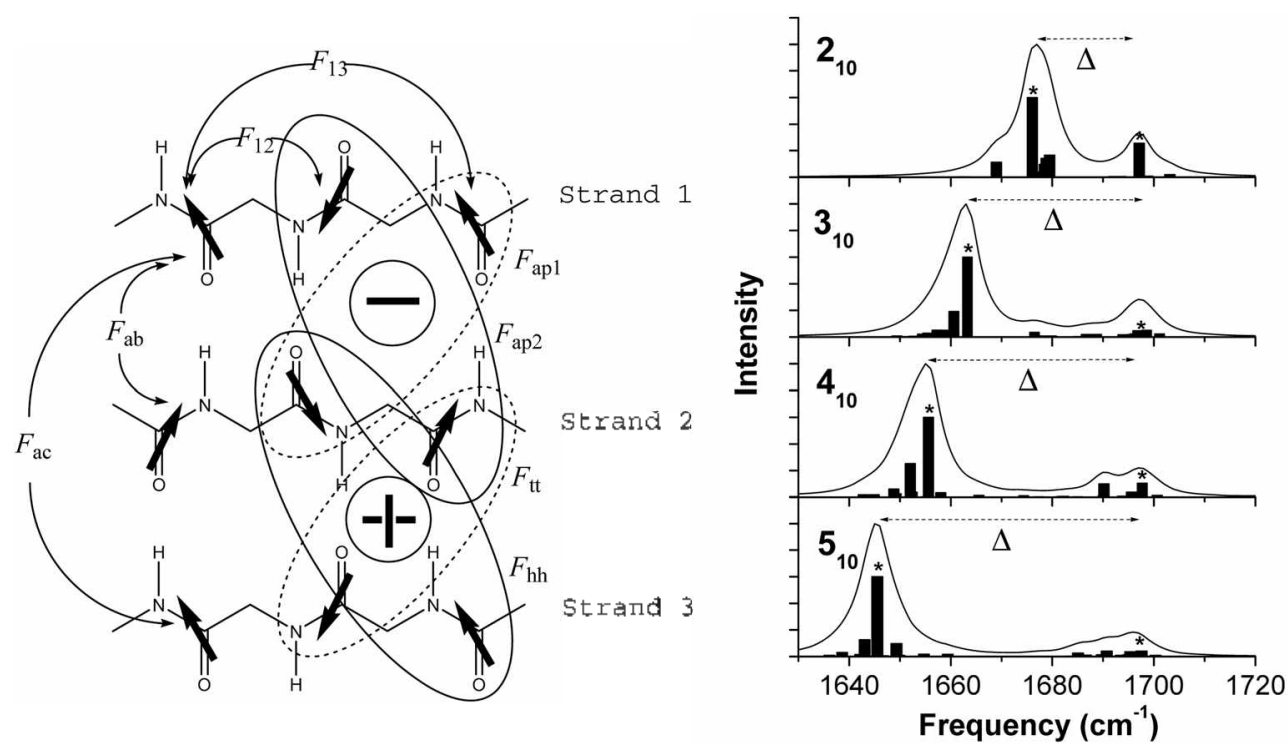

Figure 18. Different vibrational coupling constants required in the numerical simulation of vibrational spectra of anti-parallel $\beta$-sheet polypeptides. The simulated IR absorption spectra of $\mathrm{M}_{\mathrm{U}}$ anti-parallel $\beta$-sheet polypeptides, where $\mathrm{M}$ is the number of strands involved in the anti-parallel $\beta$-sheet and each strand contains 10 peptide bonds, are plotted in this figure. The frequency splitting amplitude is denoted as $\Delta$. Note that $\Delta$ increases as the number of strands, $\mathrm{M}$, increases. Adapted from [58].

correlations of fluctuating exciton state energies, we used time-correlation function formalism for the linear and nonlinear response functions associated with IR absorption and 2D IR photon echo spectroscopies. ${ }^{57}$ Numerically sinulated IR absorption and 2D IR spectra were found to be largely' deternined by the anide I normal modes delocalized on peptides in the two anti-parallel $\beta$-sheet strands (see Fig. 17). When the five peptides in the turn region are ${ }^{13} \mathrm{C}={ }^{16} \mathrm{O}$ labeled, the isotope peaks appear to be broad and featureless, whereas the $\beta$-strand region with six isotope-labeled peptides produces a well-resolved isotope peak in the IR absorption and both diagonal and cross peaks in the 2D IR spectra.

E. Anti-parallel and parallel $\beta$-sheet polypeptides. In Ref. [58]. the theoretically calculated amide 1 local mode frequencies and vibrational coupling constants in various multiple-stranded anti-parallel $\beta$-sheet polyalanines were presented and used to shed light on the spectroscopic proper- ties of these peptides with respect to the size, i.t., numbers of strands as well as of peptide bonds in a single strand. The amide I local mode frequency was found to be strongly dependent on the number of hydrogen bonds to the peptides Vibrational couplings among amide I local modes in the multiple-stranded $\beta$-sheets were shown to be fully characterized by eight different coupling constants (see Fig. 18). ${ }^{58}$ Furthemore, the intra-strand coupling constants were found to be much smaller than the inter-strand ones. By calculating the inverse participation ratio and phase-correlation factor. the extent of two-dimensional delocalization and vibrational phase relationship of anide I normal modes were elucidated. We showed that the $A \cdot E_{1}$ frequency splitting magnitude was shown to be strongly dependent on the number of strands but not on the length of each strand. A reduced one-dimensional Frenkel exciton model was therefore used to describe the observed $A-E_{1}$ frequency splitting phenomenon.

On the right-hand side of Fig. 18, the amide I IR bands of 

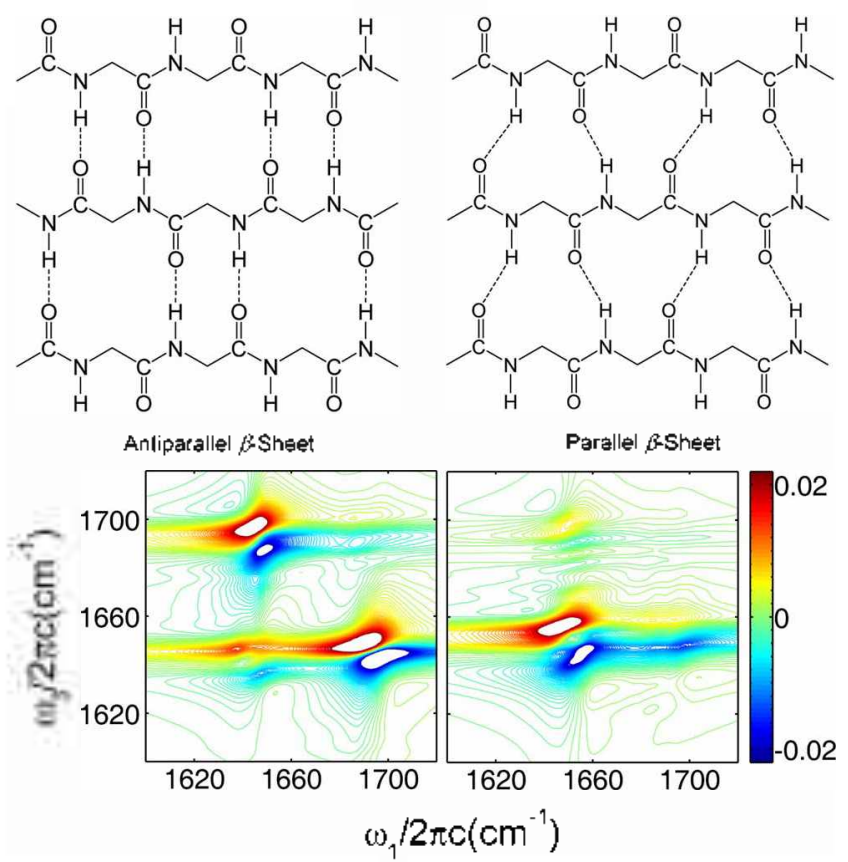

Figure 19. Molecular structures of anti-parallel and parallel $\beta$ sheets. The 2D difference spectra, defined as $S^{d i f f(T)}=S^{z Z Z Z}(T)-$ $3 S^{2 K 12}(T)$, for these two $\beta$-sheets are plotted.

$\mathrm{M}_{10}$ anti-parallel $\beta$-sheet polyalanines. where $\mathrm{M}$ is the number of involved strands. are plotted and the frequency splitting magnitude $D$ increases as $M$ increases. It was found that can be successfully fitted by using the following stretched-exponentially rising function. ${ }^{58}$

$$
\Delta(M)=\Delta(\infty)-(\Delta(\infty)-\Delta(2)) \exp \left\{-k(M-2)^{\prime /}\right\} .
$$

In the case of this model system, the parameters in Eq. (49) were estinated to be $\Delta(\infty)=57 \mathrm{~cm}^{-1}, \Delta(2)=20 \mathrm{~cm}^{-1}, k=$ 0.47 , and $\alpha=1.17$. Although the model anti-parallel $\beta$-sheet polyalanines considered were ideal. there is likely a similar relationship between the frequency splitting magnitude and the number of strands in real anti-parallel $\beta$-sheet polypeptides

The anti-parallel and parallel $\beta$-sheets are two of the most abundant secondary structures found in proteins. Despite that various spectroscopic methods have been used to distinguish these two different secondary structures. the linear spectroscopic measurements couldn't provide incisive information for distinguishing an anti-parallel $\beta$-sheet from a parallel $\beta$-sheet. After carrying out quantum chemistry calculations and model simulations. we showed that the polarization-controlled 2D IR photon echo spectroscopy can be of critical use in distinguishing these two different $\beta$ sheets. ${ }^{\text {\& }}$ Particularly, the ratios between the diagonal peaks and the cross peaks were found to be strongly dependent on the quasi-2D array of the amide I local mode transition dipole vectors. Although the parallel- or perpendicularpolarization 2D IR photon echo spectrum of the model antiparallel $\beta$-sheet peptide was found to be different from that of the parallel $\beta$-sheet peptide. the spectral difference is negligibly small. However, it turns out that the relative amplitudes of the cross peaks in the 2D difference spectrum. which is defined as $S^{\text {diff }}(T)=S^{2 Z Z Z}(T)-3 S^{2 \backslash Y Z}(T)$, of an anti-parallel $\beta$-sheet are significantly larger than those of the diagonal peaks. whereas the cross peak amplitudes in the $2 \mathrm{D}$ difference spectrum of a parallel $\beta$-sheet are much weaker than the main diagonal peak amplitudes (see Fig. 19).

We found that assuming the excited state absorption contributions to the $2 \mathrm{D}$ difference spectrum are not strongly overlap with the ground state bleaching and stimulated emission contributions in the 2D difference spectrum. the cross peak amplitude. when the $j$ th and $k$ th modes are strongly coupled, is approximately determined by the product of the two corresponding dipole strengths and angle factor, i.e. 59

$$
S_{k j}^{d i f f}(T)=S_{k j}^{z Z Z 2}(T)-3 S_{k j}^{2 W z}(T) \propto \hat{\mu_{k}^{-}} \hat{\mu_{j}^{-}} \sin ^{2} \theta_{k j}
$$

Here, the transition dipole matrix elements associated with the vibrational transitions of the $j$ th and $k$ th modes are denoted as $\mu_{i}$ and $\mu_{k}$. The angle between the two transition dipole vectors is denoted as $\theta_{7}\left(=\vec{\mu}_{k} \cdot \vec{\mu}_{3} /\left|\vec{\mu}_{k}\right| \vec{\mu}_{3} \mid\right)$. We showed that the low frequency A-mode has a transition dipole vector that is perpendicular to that of the high-frequency $\mathrm{E}_{1}$-mode. when the $\beta$-sheet forms an anti-parallel structure. Thus. the cross peak amplitude in the 2D difference spectrum is very large (see Fig. 19). In contrast. there are no such highfrequency modes of which transition dipole vectors are large in magnitude and perpendicular to that of the low-frequency A-mode. for parallel $\beta$-sheet peptide, i.e. weak cross peaks in the 2D difference spectrum. This clearly shows why the 2D spectroscopic method is a better tool to determine the global energy minimum structure of polypeptide that cannot be easily determined by other linear vibrational spectroscopic methods such as IR or Raman.

\section{Two-dimensional Electronic Spectroscopy of Coupled Multi-chromophore Systems}

As a molecular system becomes increasingly complex. such as photosynthetic complexes. molecular aggregates of quantum dots or nanoparticles. etc. conventional optical spectroscopic methods such as time- or frequency-resolved absorption spectroscopy or spontaneous emission spectroscopy are of limited use to extract direct infonmation both on the molecular properties such as electronic couplings among chromophores and on structures. In this regard. the 2D electronic spectroscopy based on heterodyne-detected photon echo spectroscopic teclunique can provide far more detailed information and be a useful method for efficient data collection. Despite that there exist quite a number of $2 \mathrm{D}$ IR spectroscopic investigations of peptides and proteins over the last decade. only a few experimental and theoretical studies with 2D electronic spectroscopy have been reported $^{7,14,18.15}$ Nevertheless. except for the fact that the electronic cluromophore can be approsimately modeled as a twolevel șistem instead of an anharmonic oscillator, one can use the same theoretical method used for polypeptides to 
numerically calculate the $2 \mathrm{D}$ electronic spectra of coupled multi-chromophore sy'stems.

Some important characteristics of the 2D-spectrum $S^{(\hat{S})}\left((0)_{\tau} . T .()_{t}\right)$ can be appreciated by realizing that the experiment effectively compares the frequency of electronic excitations (for a fixed value of $T$ ) in the two time periods of $\tau$ and $t$. For an effective two-level system with considerable static inhomogeneous broadening. the transition frequency is widely distributed. though each individual two-level system has the same frequency in both time periods. Thus. this inhomogeneity can lead to elongation of the 2D signal along the diagonal $\left(()_{\tau}=(0) t\right)$. However. if there are dynamical processes. such as excitation transfer. solvent relaxation. structural fluctuation. etc. they can scramble the optical frequencies of the individual molecules. Which can in turn make the correlation magnitude between the frequencies in the coherence state evolution periods decrease in time $T$. and the 2D spectrum at sufficiently large $T$ should become symmetric. Transient behavior of the 2D line shape can therefore provide information on the time scale of the system-bath interaction-induced decoherence process. In fact. the slope of the diagonally elongated peak decreases from 1 at $T=0$ to 0 at $T=\infty$ if there is no truly static inhomogeneity. and was found to be linearly proportional to the transition frequency-transition frequency correlation function. i.e. ${ }^{8}$

$$
\sigma_{2 D-P E}(T)=\langle\delta \omega(T) \delta \omega(0)\rangle\left\langle\left\langle\delta \omega^{2}\right\rangle .\right.
$$

where $\sigma_{I D-P E}(T)$ is the slope of the diagonally elongated 2D photon echo contour.

The time-domain signal $S^{(j)}(\tau . T, t)$ can. in principle, be obtained from experiment based on the heterodyne-detected photon echo measurement. and its double Fourier transformation in $\tau$ and $t$ leads to $S^{i 3 i}\left(()_{\tau}, T, \omega_{t}\right)$. In Ref. [18]. when there are excitation transfers among the excited states we showed that the 2D photon echo spectnum is given by a sum of five distinctively different contributions as

$$
\begin{aligned}
S^{(3)}\left(\Omega_{1}, T . \Omega_{j}\right)= & G B+S E(j \rightarrow j)+S E(j \rightarrow k) \\
& -E A(j \rightarrow j)-E A(j \rightarrow k)
\end{aligned}
$$

where $G B . S E$. and $E A$ represent the ground-state bleaching. stimulated emission. and excited state absorption. respec- tively (see Eq. (33) in Ref. [18]). In the above equation. " $(j$ $\rightarrow k$ )" means that the initially created population state on the $j$ th one-exciton state undergoes a transition to the $k$ th state during $T$. and summations over $j$ and $k$ should be performed. Therefore. the last four terns in Eq. (52) include such population transfer processes. In the case when the excitation transfer processes are incoherent. one can solve the Master equation for conditional probability functions. i.e.

$$
\frac{d}{d t} G_{k l l}(t)=\sum_{j=k} K_{k l} G_{l l}(t)-\left(\sum_{l i k} K_{l k}\right) G_{k l}(t)
$$

Here. $G_{b l}(t)$ is the conditional probability of finding the population on the $k$ th exciton state at time $t$. when it was on the $j$ th state at time zero. The $(j \rightarrow k)$ transition rate constant was denoted as $K_{k 7}$. As shown earlier. the transition rate constant between two one-exciton states is linearly proportional to the spatial overlap between $\left.|\psi|\right|^{2}$ and $\left|\psi_{k}\right|^{2}$. i.e.

$$
K_{k_{l}} \propto\left\langle\delta \Omega_{l} \delta \Omega_{k}\right\rangle .
$$

It should be noted that $\left\langle\delta \Omega_{j} \delta \Omega_{k}\right\rangle$ is the correlation magnitude between the two fluctuating transition frequencies and in principle is a measurable quantity by using the two-color photon echo peak shift measurement method (see Eq. (43)).

Now let us consider off diagonal (cross) peaks and their plysical meanings. These peaks arise only when the electronic states of the individual molecules comprising the complex interact (mix) and their amplitudes and locations provide extra infomation on the molecular structure and coupling strength of the coupled multi-chromophore system. Although the detailed theoretical expression for the cross peak in a given 2D spectrum is highly complicated due to the multi-dimensional line-broadening term. we found that the amplitude of the cross peak $S_{i 7}(t)$ at $\left(\omega_{r}=\Omega_{r}, \omega_{r}=\Omega_{k}\right)$ is approximately proportional to the product of the associated conditional probability function and transition dipole factors i.e.

$$
S_{k j}(T) \propto G_{k j}(t)\left[\left\langle\mu_{g k}^{2} \mu_{g l}^{2}\right\rangle-\left\langle\mu_{g i} \mu_{k \rightarrow j}^{2} \mu_{g l}\right\rangle\right]
$$

where $\mu_{g k}$ is the transition dipole from the ground to the $k^{\text {th }}$ one-exciton state and $\mu_{k f}$ from the $k^{\text {th }}$ one-exciton state to the $f^{\text {th }}$ two-exciton state and $\omega_{k j}=\omega_{g k}$. Eq. (55) shows that the appearance and disappearance of cross peaks are directly
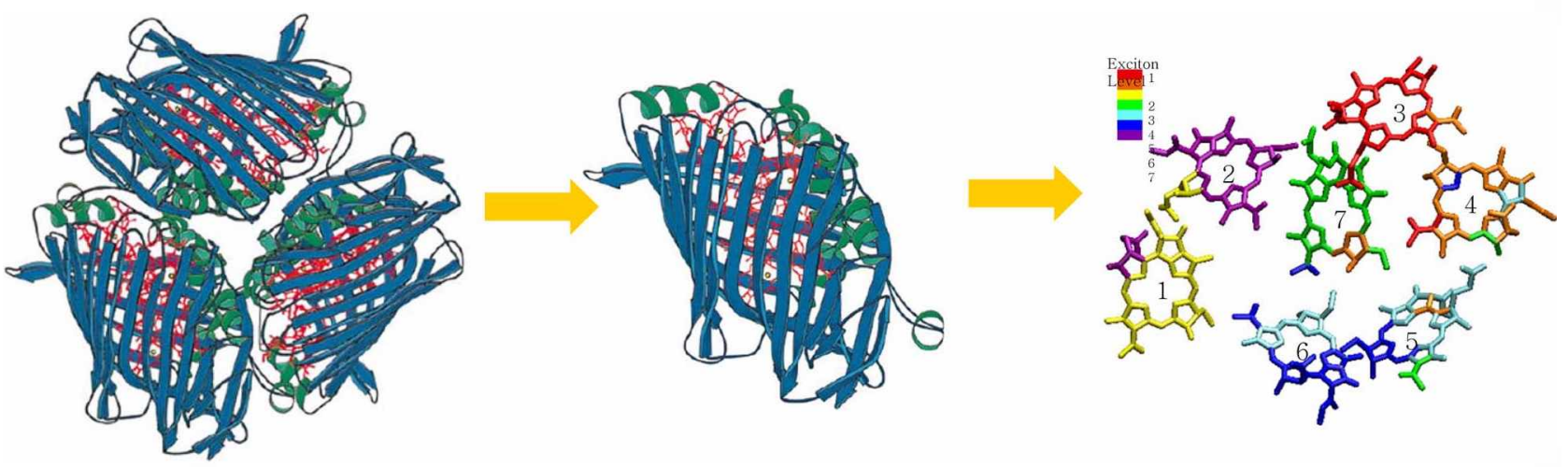

Figure 20. FMO light-harvesting protein complex consists of three protein subunits. Inside the $\beta$-sheet barrel, there exist seven $\mathrm{BChl}$ 's. Different color in the last figure represents its relative energy level. 


\section{QUANTUM INTERFERENCE}

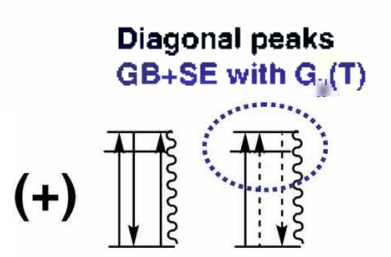

Off-diagonal peaks GB
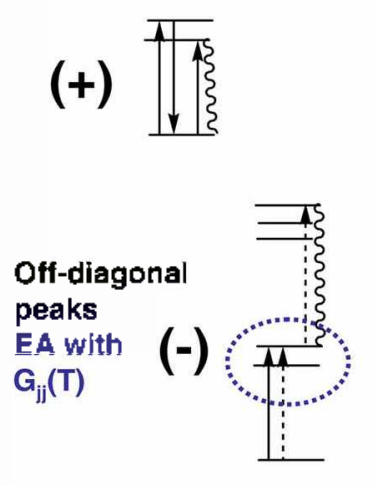

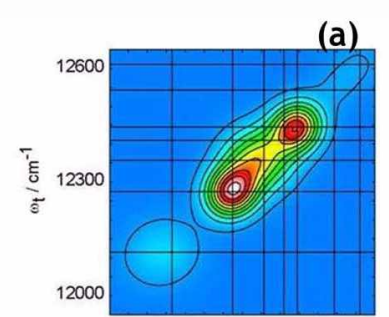

(b)

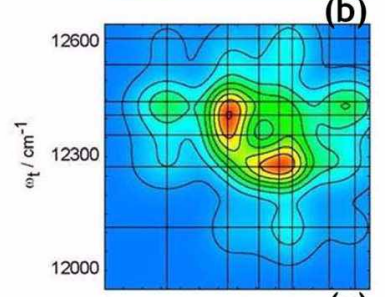

(c)

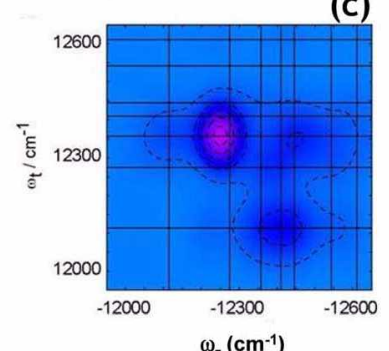

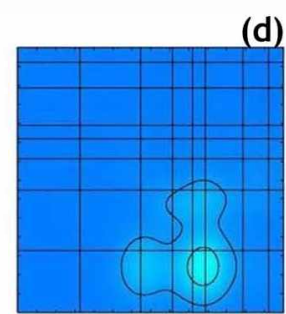

(d)
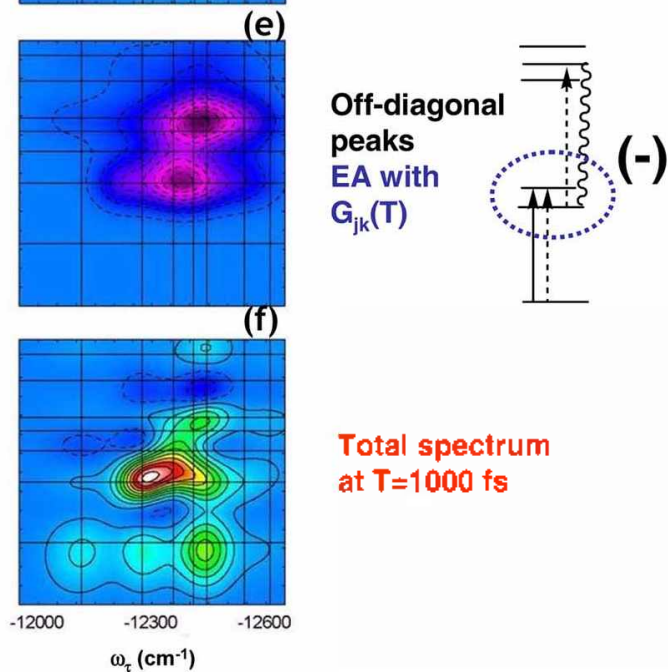

Off-diagonal peaks

SE with $\mathrm{G}_{\mathrm{jk}}(\mathrm{T})$

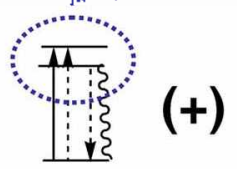

Total spectrum

at $T=1000$ fs

Figure 21. Numerically simulated 2D electronic spectrum of FMO complex is plotted in Figure (f), where the waiting time $T$ is 1 ps. Five different sets of nonlinear optical transition pathways produce distinctively different 2D spectra (figures (a)-(e)). Adding these five spectra gives the total 2D spectrum in figure (f). Adapted from [18].

related to the excitation transfer within the one-exciton state manifold. This shows that the 2D spectroscopy is a powerful method to delineate the complicated kinetic network among coupled chromophores.

FMO complex. Recently. Tobias et al. perfomed 2D photon echo experiments on the Fenna-Mathews-Olson (FMO) complex consisting of seven bacteriochlorophỵll molecules (see Fig. 20).

In Ref. $[18]$. a detailed theoretical description of the timeevolved 2D photon echo spectra and deduced excitation transfer mechanism were presented. Using the theoretical model developed for this FMO complex. we numerically simulated the $2 \mathrm{D}$ spectra and plot the $1 \mathrm{ps}$ snapshot spectrum in Figure 2l(f). In addition to the main diagonal peaks that are directly associated with the peaks in the absorption spectrum. several cross peaks are clearly observed. The total 2D spectrum is essentially a sum of five different sets of nonlinear optical transition pathways so that the quantum interference at the amplitude level is critical in understanding the detailed 2D line shape. We calculated these contributions separately and plotted them in Figures 21(a)-(e). Adding these five spectra to get the total spectrum in Figure 2l(f) means that these different quantum pathways constructively or destructively interfere to produce the total 2D spectrum. The cross peak amplitudes in the bottom half region of Figure 2l(f) were observed to increase in time and their time-dependent changes were shown to directly provide state-to-state population transfer rates and pathways.

In Figure 21, we also plot the representative energy level diagrams. The Figures 21(a). (b). and (d) are associated with the GB and SE contributions and they all are positive. If one ignores the population transfer processes completely and considers the GB and SE tenns. the 2D spectnum would appear approximately diagonally symmetric in shape. However. due to the existence of population transfer processes in the one-exciton state manifold and of the EA contribution (see Figs. 2l(c) and 21(e)), the total 2D electronic spectrum in Figure 21(f) becomes highly asymmetric. Nevertheless. the lower part of the spectrum in Figure 21(f) is largely dictated by the SE involving population transfer from upperlying one-exciton states to lower-lying states. As $T$ increases. not only the cross peak amplitudes but also the amplitude and detailed contour line shapes of diagonal peaks change in a very complicated way. but they can be quantitatively described theoretically once the electronic couplings and site energies are accurately determined.

Dimer. Although we have discussed a rather complex molecular system. e.g. FMO light-harvesting protein. one can leann a great deal of underlying physics of $2 \mathrm{D}$ spectroscopy by considering a simple excitonically coupled dimer system. Here. again each monomer is modeled as a twolevel system so that for this dimer sy'stem there are two oneexciton states $\left|e_{\mid}\right\rangle$and $\left|e_{2}\right\rangle$, and a single two-exciton state $|f\rangle$. When the population transfer between the two one- 
exciton states is relatively slow and when the two local transition dipoles $d_{1}$ and $d_{2}$ have the same magnitudes. i.e. $d=d_{3}=d_{2}$. one can estinate the maximum (or minimum) amplitudes of the diagonal and cross peaks. denoted by $S_{j h}^{j}$. $\operatorname{as}^{34}$

(1) Diagonal peak at $\left(\omega_{1}=\bar{\omega}_{\varepsilon_{1}}, \omega_{3}=\bar{\omega}_{e_{1} g}\right)$

$$
S_{11}^{\hat{\prime}}=\frac{2 \pi}{\left\langle\delta \Omega_{1}^{2}\right\rangle}\left\{2\left\langle\mu_{e_{1}}^{2} \mu_{e_{1}}^{2}\right\rangle\right\}=\frac{2 \pi|d|^{+}}{\left\langle\delta \Omega_{1}^{2}\right\rangle}\left\{12(1+2 k \cos \phi)^{2}\right\}
$$

(2) Diagonal peak at ( $\left.\omega_{1}=\bar{\omega}_{\varepsilon_{\wedge} g}, \omega_{3}=\bar{\omega}_{e_{\wedge} g}\right)$

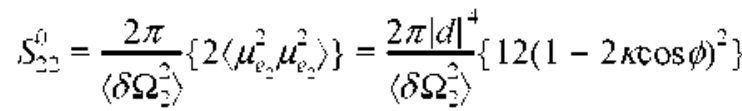

(3) Off-diagonal peak at $\left(\omega_{1}=\bar{\theta}_{\varepsilon_{1}, g} \cdot \omega_{\hat{3}}=\bar{\phi}_{\xi_{3} g}\right)$

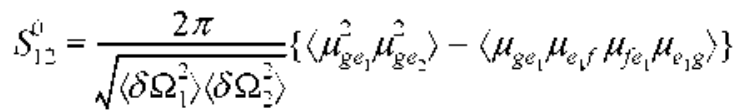

$$
\begin{aligned}
& =-\frac{2 \pi|d|^{4}}{\sqrt{\left\langle\delta \Omega_{1}^{2}\right\rangle\left\langle\delta \Omega_{3}^{2}\right\rangle}}\left\{8\left(2 \kappa^{2}+3 \kappa \cos \phi+4 \kappa^{2} \cos ^{2} \phi\right)\right\}
\end{aligned}
$$

(4) Off-diagonal peak at $\left(\omega_{1}=\bar{\omega}_{\ell_{2} g .} \omega_{3}=\bar{\omega}_{\iota_{1} g}\right)$

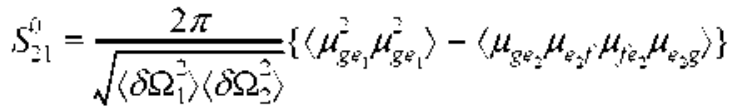

$$
\begin{aligned}
& =-\frac{2 \pi|d|^{+}}{\sqrt{\left\langle\delta \Omega_{1}^{2}\right\rangle\left\langle\delta \Omega_{\Omega}^{2}\right\rangle}}\left\{8\left(2 k^{2}-3 k \cos \phi+4 k^{2} \cos ^{2} \phi\right)\right\} \text {. }
\end{aligned}
$$

Here, $\kappa$ is a measure of the delocalization of the excited states and is defined as

$$
\kappa \equiv \cos \theta \sin \theta \text {. }
$$

where the mixing angle $\theta$ is determined as $\tan 2 \theta=2 J_{1} /$ $\left(\omega_{1}-()_{2}\right)$. The electronic coupling constant between the two monomers is $J_{12}$ and the electronic transition frequencies of the two monomers are $\omega_{1}$ and $\omega_{s}$. The angle between the two monomer transition dipole vectors is $\phi$.

Note that the cross peak amplitude $S_{12}^{j}$ is given by the difference between $\left\langle\mu_{g \varepsilon_{1}}^{2}, \mu_{g \varepsilon_{2}}^{2}\right\rangle$ and $\left\langle\mu_{g \varepsilon_{1}} \mu_{\varepsilon_{1}+} \mu_{f \varepsilon_{1}} \mu_{\varepsilon_{1} g}\right\rangle$. That is to say, the two different transition pathways interfere to produce the cross peak. However, these two terms exactly cancel out only when the two monomers do not electronically interact. Therefore. the existence of cross peaks is definite evidence of non-zero electronic coupling. It is also interesting to note that the cross peak amplitude can provide critical information on the relative orientation of the two monomers because it is a function of $\phi$.

Overall. it was demonstrated that the 2D optical spectroscopy enables couplings. relaxation pathways and rates. and spatial relationships between exciton states to be measured. Also, by tracking the cross peak amplitudes in time. one can direct follow the energy flow on a molecular length scale with fentosecond time resolution. Thus. two-dimensional electronic spectroscopy should provide insights into all systems with electronic band structures.

\section{Perspectives and Concluding Remarks}

Ultrafast 2D vibrational and electronic spectroscopies have been paid a lot of attention because they can provide detailed and highly dense information on molecular structure of peptides and proteins. molecular interactions and dynamics. nucleic acid structures. and semiconductor dynamics. One of the most important advantages of this spectroscopic teclinique is its unprecedented ultrafast time-resolution so that it will trigger a number of new researches and investigations of chemical reaction dynamics and biochemical processes involving transient species. Conventional applications of time-resolved spectroscopy such as pump-probe method mainly focus on measurements of life-times of an excited state and of radiationless transition rates among different quantum states. In order to elucidate the entire kinetic network and rates. one should perform quite a number of independent two-color pump-probe measurements by varying pump and probe field frequencies separately. That can be quite tedious and time-consuming. Furthermore due to time-energy uncertainty, it is not possible to achieve both ultrafast timeresolution and high frequency-resolution simultaneously. On the other hand. since the 2D spectroscopy utilizes femtosecond laser pulses with broad spectral bandwidths, colerent quantum states of which energies are within the pulse spectral bandwidth can be created simultaneously and probed in time by using yet another femtosecond laser pulses. In this section, we will discuss various possible applications of $2 \mathrm{D}$ spectroscopy and some perspectives. and finaly present a few concluding remarks.

A. Chemical reaction dynamics. One of the most important applications of ultrafast 2D spectroscopy would be to study chemical reaction dỵanics in condensed phases includ-ling solutions. surfaces. and interfaces. The spatial connectivity between any two different vibrational (or electronic) degrees of freedom (chromophores) via throughbond or through-space interactions is the key information that can be extracted from the time-resolved multidimensional spectra. Thus in general by properly selecting two vibrational (electronic) degrees of freedom directly associated with reactive species. one can in principle follow the chemical reaction dynamics by monitoring the cross peak amplitude changes in time.

Intramolecular vibrational energy redistribution: The first phenomenon closely related to the chemical reaction dynamics is the intramolecular vibrational energy redistribution process. It was shown that ultrafast IR-Raman spectroscopy is a useful method for such an investigation. but its difficulty in detecting the weak incoherent Raman scattering signal is an obstacle limiting its wide applications for studying IVR process of reactive species in condensed phases. Ultrafast multi-dimensional vibrational spectroscopy detecting the coherent electric field might be of use in the future. For instance, dual frequency 2D IR pump-probe or photon echo methods can be used to following state-to-state IVR pathways by examining the temporal evolution of cross peaks. If the pump and probe field frequencies are indepen- 
dently tunable and the probe pulse is sufficiently short enough to cover a large range of vibrational excited states. the measured cross peak amplitudes can provide information on the population transfer from the initially excited state to probing states.

Solvent role in a chemical reaction: The role of the solvent molecules in chemical reactions has been the central research theme of physical chemistry. In addition. the solvent plays a critical role in stabilizing protein structure in its native conformation. However. there is still a lack of experimental method to obtain direct information on how each individual solvent molecule participates in a given chemical reaction. Most of previous spectroscopic investigations were to follow the dynamics and spectral changes of probing solute mode to indirectly infer an unknown timescale and dynamics of interacting solvent molecules. Now: it could be possible to use the ultrafast 2D spectroscopy to simultaneously follow the dynamics of both solute and solvent modes. Suppose that there is a characteristic vibrational chromophore in reactive species and that its vibrational properties such as frequency and dipole strength are strongly coupled to a particular solvent vibrational motion. These two mode frequencies are now denoted as $\omega_{\text {solute }}$ and $\omega_{\text {solvent. }}$ If the laser pulse frequencies, $(i)$ and $\omega_{2}$. are tuned to

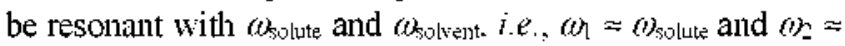
$\omega_{\text {solvent. }}$ only when the two characteristic modes are vibrationally coupled to each other does the $2 \mathrm{D}$ vibrational spectroscopic signal, i.e. cross peaks. not vanish. The cross peak at $\left(()_{1} \approx \omega_{\text {solute, }}()_{-} \approx()_{\text {solvent }}\right)$ is therefore a direct indicator revealing how the solvent mode is participated in the course of this chemical reaction. Two-dimensionally displayed spectra in time will thus give a detailed picture on the solvent dynamics during the chemical reaction. A few potentially interesting chemical reactions are photo-dissociation. photo-induced electron transfer. excited state isomerization. proton transfer induced by a photo-dissociation or photoexcitation. etc.

Chemical exchange: The 2D spectroscopy has been shown to be exceptionally useful in studying hydrogen-bond formation and dissociation processes and van der Waals complexation dy'namics. An advantage of this application is that this teclunique does not need any external ultrafast perturbation such as T-jump. pressure-jump. concentration change. etc. Note that the cross peak amplitude changes in time are directly related to the population changes of chemical species (reactants) initially pumped at time zero to product species of which characteristic mode frequencies are in different spectral (probing) window. However. thus far the 2D spectroscopic teclunique has been used to study relatively weak intermolecular interaction such as $\mathrm{H}$-bonding and dispersion interactions. One can however use this method to directly follow the chemical reaction dynamics by examining the cross peak. where the two chromophores are specifically belonging to reactants and products exclusively. Then. may be it will be possible to follow the entire kinetic network even for chemical reactions with multiple intermediates in femtosecond time scale.
B. Solvation structure and dynamics. Solvation dynamics has been extensively studied over the last two decades. Among different experimental methods. the fluorescence Stokes shift measurement has been one of the most effective methods. The other widely used method is the photon echo peak shift (PEPS) measurement. PEPS was shown to be useful not only to study ultrafast solvation dynamics but also to quantitatively measure the inhomogeneous width. Nevertheless. it is still desired to have an experimental method providing direct information on how the surrounding solvent molecules participate in the solvation dynamics and in the formation of the local structure around it. The IR pumpprobe and photon echo methods have shown to be useful for such a purpose. However. these works have focused on the vibrational dynamics of the solute only that is to say the solute-solvent interaction-induced changes of the vibrational properties of the solute (not both solute and solvent) were only observed.

Then. are there any experimental methods that can be used to study solvation dynamics by watching the vibrational dynamics of both solute and solvent? As emphasized in this review. 2D vibrational spectroscopy can provide direct information on the coupling between two spatially separated but coupled vibrational modes. Let's assume that the two external field frequencies. which are either IR frequency or stimulated Raman vibrational frequency. are adjusted to be resonant with the two vibrational chromophores. when one of the two belongs to the solute and the other to the solvent (see the discussion in the above subsection). Then. the timedependent change of the cross peak amplitude will give us direct information on the solute-solvent interaction. i.e.. microscopic aspect of the solvation dynamics.

C. Vibrational interactions between adsorbed molecules: Surface science. Vibrational dynamics of adsorbed molecules on surfaces or at interfaces has been studied by using IR reflection. IV-SFG (IR-vis sum-frequency-generation) spectroscopy. etc. However. previous experimental methods are essentially one-dimensional spectroscopy so that only one type of vibrational chromophore is under direct investigation at a time. Therefore. $2 \mathrm{D}$ or $3 \mathrm{D}$ vibrational spectroscopy specifically designed for molecular systems on surfaces or at interfaces will be of critical use in studying vibrational interactions among different chemical species on surfaces. For instance the IR-IR SFG or DFG (difference-frequencygeneration) method can be used to study vibrational coupling between two different adsorbates. Once the experimental sensitivity issue is overcome, the IR-IR SFG (DFG) or other surface $2 \mathrm{D}$ vibrational spectroscopies will open up a new research area in surface science. Furthermore. one can use surface 2D vibrational spectroscopy for the investigation of inhomogeneous catalysis. surface melting. wetting. and confinement-induced phase transition, etc.

D. Biological applications. One of the most important applications of the ultrafast multidimensional vibrational spectroscopy could be to biological science.

Structure determination of protein: The 2D IR spectroscopy has already been shown to be a useful method for 
determining local structure of peptides. Other types of 2D vibrational spectroscopies and dual frequency IR photon echo spectroscopies will serve as critical tools for this purpose. In addition. one can combine vibrational and electronic transition processes. e.g. triply resonant $2 \mathrm{D}$ vibrational spectroscopy or vibrational/electronic four-wave-mixing spectroscopy. The correlation between two modes that are coupled to both vibrational and electronic transitions of the chromophore. such as a peptide bond is likely to be sensitive to the 3D structure of the polypeptide backbone.

Alternatively: the electronically resonant fifth-order Raman spectroscopy and fifth-order three pulse scattering spectroscopy might be of use to get information on the 3D structure of proteins. Suppose the two optical field frequencies are simultaneously resonant with two electronic chromophores. such as phenol ring in Tyr and peptide bond in a given protein. Dual frequency 2D electronic spectroscopy could give us quantitative information on coupling between these two distinctively different electronic transitions. which enable 3D structure of polypeptides to be determined.

Protein folding dynamics: As emphasized in this review and by others. the key advantage of the ultrafast multidimensional spectroscopy is its experimentally accessible time-scale. Although the protein folding occurs in a wide range of time scales. from picoseconds to seconds. solution NMR cannot be used to study the early part of the protein folding process due to its limited time resolution. In this respect. the ultrafast multi-dimensional spectroscopy utilizing IR or visible pulses has a clear advantage over the other tecluniques. Recently. the nonlinear spectroscopy utilizing circularly polarized beams was theoretically proposed and is expected to be useful for studying protein folding dynamics since it is an ultrafast optical activity measurement technique.

Enzyme catalysis and protein-ligand binding process: Another interesting application of the ultrafast multi-dimensional vibrational spectroscopy is to investigate the substrate-enzyme interaction. In order for a given enzyme to catalyze the biochemical reaction. the substrate (or ligand) should form a complex with the catalytic site of the enzrme. Tuning the two external field frequencies to be in resonance with the characteristic vibrational modes of the substrate and enzyme. one can directly measure the formation and dissociation of the ES complex. Similarly. protein-DNA (or proteinRNA) complexes. antibody-antigen complexes etc can be other interesting targets to be studied with the ultrafast multi-dimensional spectroscopy.

In this review, we have summarized our recent theoretical investigation results demonstrating a wide range of applications of coherent multi-dimensional optical spectroscopy. It is believed that this relatively young spectroscopic method has a great potential and is highly useful in studying chemical and biological processes in real time.

Acknowledgments. This work was supported by the creative research initiatives program of KOSEF (MOST. Korea). The author thanks graduate students. post-doctoral research associates at the Center for Multidimensional Spectroscopy. Korea University. and many other collaborators.

\section{References}

1. Hamm. P.: Lim, M: DeGrado, W. F.: Hochstrasser, R. M. Proc. Natl. Acad Sci. USA 1999. 96.2036.

2. Khalil. M:: Demirdövet1. N.: Tokmakoff. A. J. Phos Chent. A 2003. 107. 5258 .

3. Wouterset1. S.: Hamm. P. J. Phrs Cond Matt 2002. 1t. R1035.

4. Wright. J. C. Int Rev Phys Chem 2002, 21, 185.

5. Cho, M. In Adrances in Ifulti-photon Processes and Spectroscopv: Lin. S. H; Villaeys. A. A.: Fujimura, Y.. Eds.: World Scientific: Singapore. 1999: Vol. 12. p 229.

6. Mukamel. S. Amm. Rev: Phys. Chent. 2000.51.691

7. Jonas. D. M. Amm. Rev Phns Chent 2003. 54. 425.

8. Kwac. K.: Cho, M. J. Plys Chem. A 2003, 107, 5903.

9. Woutersen, S:; Mu, Y; Stock, G.; Hamm. P. Chem. Plys. 2001. 266.137.

10. Kwac. K.: Lee. H.: Cho. M. J. Chem. Phs 2004. 120. 1477.

11. Kwac. K.: Cho. M. J. Raman Spectrose. 2005 . 36. 326.

12. Kim. Y. S.: Hochstrasser. R. M. Proc. Natl Acad Sci. US.A. 2005. 102,11185 .

13. Zheng. J.: Kwak, K.; Asbury, J.; Chen. X.: Piletic. I. R.: Fayer, M. D. Science $2005.309,1338$.

14. Brinter. T.: Stenger. T.: Vaswami. H.: Cho. M.: Blankenship. E.: Fleming. G. R. Nature $2005 .+3+.625$

15. Wuthrich. K. MIR of Proteins and Nucleic Acids: WileyInterscience: New York. 1986

16. Ernst. R: Bodenhausen, G.: Wokaun. A. Principles of Xuclear Magnetic Resonance in One and Two Dintensions: Clarendon: Oxford. 1987.

17. Sanders. T. K. M.: Hunter. B. K. Modem MIR Spectroscopy: Onford University Press: New York. 1994.

18. Cho. M.: Vaswani. H. M.: Brinter. T.: Stenger. J.: Flening. G. R. J. Phus. Chem. B 2005, 109. 10542

19. Cho. M: Brixner, T.; Stiopkin. I.: Vaswani. H.; Fleming. G. R. $d$. Chinese Chem Soc, 2006, 53,15

20. Cho. M. Phws.hentContm. 2012. 5. 40.

21. Cho. M. J. Chent. Phis. 2002. 116.1562

22. Cho. M. J. Chent. Phys 2003. 119. 7003

23. Cheon. S.: Cho, M. Phus. Rev A $2005,71.013808$

24. Fleming. G. R. Cho. M. Amu Rev Plys. Chem. 1996. 47, 103.

25. Wiersma. D. A.: de Boeij, W. P. Pshenichnikov, M. S. Amut Rev Phus Chem. 1998. +9.99.

26. Mukamel. S. Principles of Nonlinear Optical Spectroscopy: Onford University Press: New York. 1995.

27. Sung. J.: Cho, M. J. Chem. Plys. 2000. 113,7072

28. Sung. J.: Silbey. R. J.: Cho. M. J. Chem. Plys. 2001. 115. 1422.

29. Cho. M. J. Chom Phys. 2001. 115. 424 .

30. Zhang. W. M.: Meier. T.: Chernyak. V: Mukamel. S. J. Chent. Phs 1998. J08.7763.

31. (a) Yantg. M.: Fleming. G. R. J. Chent. Ptns. 1999, 110, 2983. (b) Agarwal, R: Prall. B. S.: Rizvi, A. H.; Yang. M.: Fleming. G. R. . Chent. Phis. 2002. 116. 6243. (c) Prall. B. S.: Parkinsont. D. Y.: Fleming. G. R.: Yang, M; Ishikawa, N. d. Chem. Plys. 2004. 120. 2537.

32. Kobayashi. T. J-aggregates: World Scientific: Singapore. 1996.

33. Cho. M.: Yu. T.-Y.: Too. T: Nagasawa. Y.: Passino. S. A.: Fleming. G. R. J. Plws. Chem. 1996, 100. 11944

34. Cho. M. Fleming, G. R. J. Chem. Phus. 2005, 123, 114506.

35. Ham. S.: Kim. J.-H.: Lee. H.: Cho. M. J. Chem Plys. 2003. 118. 3491.

36. Cho. M. J. Chen. Phws 2003. 118.3480

37. Eaton. G.: Symons. M. C. R. J. Chem. Soc. Farador Trams 1989. 85.3257.

38. Kwac. K; Cho, M. J. Chem. Phys. 2003, 119, 2247. 
39. Woutersen. S.: Pfister. R.: Hamm. P.: Mu. Y. G.: Kosov. D. S: Stock. G. J. Chem. Phns. 2002. H7. 6833.

40. Zanni. M. T.: Asplund. M. C.: Hochstrasser. R. M. J. Chent. Phus. 2001. H. 4579

41. DeCamp, M. F: DeFlores. L.: MeCracken. I. M: Tokmakoff A.: Kwac, K: Cho. M. J. Phys. Chem. B 2005. 109. 11016.

42. Kwac K. Cho. M. J. Chem. Phvs. 2003. 119, 2256

43. Infrared Spectroscopy of Biomolecules: Mantsch. H. H.: Chapman. D. Eds.: Wiley-Liss: New York. 1996.

44. Infrared and Raman Spectroscopy of Biological Materials: Gremlich. H.-U.: Yan, B., Eds:: Mareel Dekker: New York: 2000.

45. Cinutar Dichroism: Principles and Applications: Berova. N.: Nakanishi. K.: Woody. R. W. Eds.: Wiley-Liss: New York. 2000.

46. Eker. F.: Griebenow: K.: Schweitzer-Stenner. R. J. Ain. Chem. Soc. 2003.125 .8178$.

47. Han, W.-G.: Talkanen. K. J.; Elstner. M.: Suhai, S. J. Phys. Chem. B 1998. 102.2587.

48. Drozdov. A. N.: Grossfield. A.: Pappu, R. V. J. Am. Chem. Soc.
2004. 126,2574 .

49. Zanni. M. T.: Gnanakaran. S.: Stenger. J.: Hochstrasser. R. M. J. Pho Chen. $B$ 2001. 105.6520.

50. Ge. N.-H.: Zanni. M. T.: Hochstrasser. R. M. J. Phns. Chent. A 2002. 106,962 .

51. Rubtsov, I. V: Hochstrasser, R. M. J. Phys. Chem. B 2002. 106. 9165.

52. Haht1. S.: Lee. H.: Cho. M. J. Chem. Phws 2004. 121. 1849.

53. Lee. K.-K.: Hahn. S.: Oh. K.-I.: Choi. J. S.: Ioo. C.: Lee. H.: Han1. H.: Cho. M. J. Phws Chem. B 2006. in press.

54. Ham. S.: Hahn, S.: Lee, C: Kim, T.-K.: Kwak. K: Cho, M. $d$ Phus. Chem. B 2004, 108, 9333.

55. Woutersen. S.: Hamm. P. J. Chen. Phus 2001. M5. 7737.

56. Choi. T. H.: Hahnt. S.: Cho. M. Int. J. Onantm Chem. 2005. 104. 616.

57. Hahn1. S.: Ham. S.: Cho. M. J. Phs Chent B 2005. 109. 11789.

58. Lee. $\mathrm{C}$ : Cho, M. J. Phws. Chem. B 2004, 108, 20397.

59. Hahn. S.; Kim. S.-S.: Lee, C.: Cho, M. J. Chem. Plns. 2005.123. 0884905. 